\title{
Generalized Repeated Interaction Model and Transfer Functions
}

\author{
Santanu Dey and Kalpesh J. Haria
}

\begin{abstract}
Using a scheme involving a lifting of a row contraction we introduce a toy model of repeated interactions between quantum systems. In this model there is an outgoing Cuntz scattering system involving two wandering subspaces. We associate to this model an input/output linear system which leads to a transfer function. This transfer function is a multi-analytic operator, and we show that it is inner if we assume that the system is observable. Finally it is established that transfer functions coincide with characteristic functions of associated liftings.
\end{abstract}

Mathematics Subject Classification (2010). Primary 47A13; Secondary 47A20, 46L53, 47A48, 47A40, 81R15.

Keywords. repeated interaction, quantum system, multivariate operator theory, row contraction, contractive lifting, outgoing Cuntz scattering system, transfer function, multi-analytic operator, input-output formalism, linear system, observability, scattering theory, characteristic function.

\section{Introduction}

In page 287 of the article 9] the author has commented the following while comparing [9] with [4, 5]: In [4] a row contraction $\underline{A}$ on a Hilbert space $\mathcal{H}$ with a one-dimensional eigenspace is considered and the theory of minimal isometric dilations is used. The characteristic function introduced in [5] is a multi-analytic operator associated to a lifting and the ergodic case is studied in detail in 4]. In 9] minimality is not considered but one starts with an interaction $U$ (which is a unitary operator) in a scheme similar to [4] and and obtains a multi-analytic operator which represents the transfer function of an input-output system associated with the interaction. It is expected that the scheme developed [9] is more directly applicable to physical models. In the setting of [5] the assumption of a one-dimensional eigenspace is dropped and the theory is much more general in another direction. A further integration of these schemes in the future may help to remove unnecessarily restrictive 
assumptions of the toy model considered in [9] and lead to the study of other and of more realistic models.

This paper achieves some of these objectives. In the model of repeated interactions between quantum systems, also called a noncommutative Markov chain, studied in 9 (cf. 8) for given three Hilbert spaces $\mathcal{H}, \mathcal{K}$ and $\mathcal{P}$ with unit vectors $\Omega^{\mathcal{H}}, \Omega^{\mathcal{K}}$ and $\Omega^{\mathcal{P}}$ an interaction is defined to be a unitary operator $U: \mathcal{H} \otimes \mathcal{K} \rightarrow \mathcal{H} \otimes \mathcal{P}$ such that

$$
U\left(\Omega^{\mathcal{H}} \otimes \Omega^{\mathcal{K}}\right)=\Omega^{\mathcal{H}} \otimes \Omega^{\mathcal{P}} .
$$

Define $\mathcal{K}_{\infty}:=\bigotimes_{i=1}^{\infty} \mathcal{K}$ and $\mathcal{P}_{\infty}:=\bigotimes_{i=1}^{\infty} \mathcal{P}$ as infinite tensor products of Hilbert spaces with distinguished unit vectors. We denote $m$-th copy of $\mathcal{K}$ in $\mathcal{K}_{\infty}$ by $\mathcal{K}_{m}$ and set $\mathcal{K}_{[m, n]}:=\mathcal{K}_{m} \otimes \cdots \otimes \mathcal{K}_{n}$ for $m \leq n$. Similar notations are also used with respect to $\mathcal{P}$. The repeated interaction is defined as

$$
U(n):=U_{n} \ldots U_{1}: \mathcal{H} \otimes \mathcal{K}_{\infty} \rightarrow \mathcal{H} \otimes \mathcal{P}_{[1, n]} \otimes \mathcal{K}_{[n+1, \infty)}
$$

where $U_{i}$ 's are copies of $U$ on the factors $\mathcal{H} \otimes \mathcal{K}_{i}$ of the infinite tensor products and $U_{i}$ 's leaves other factors fixed. Equation (1.1) tells us that the tensor product of the vacuum vectors $\Omega^{\mathcal{H}}, \Omega^{\mathcal{K}}$ (along with $\Omega^{\mathcal{P}}$ ) represents a state of the coupled system which is not affected by the interaction $U$. This entire setting represents interactions of an atom with light beams or fields. In particular $\Omega^{\mathcal{H}}$ in $\left[9\right.$ ] is thought of as the vacuum state of an atom, and $\Omega^{\mathcal{K}}$ and $\Omega^{\mathcal{P}}$ as a state indicating the absence of photons.

In the generalized repeated interaction model that we introduce in this article we use a pair of unitaries to encode the interactions instead of one unitary as follows:

Let $\tilde{\mathcal{H}}$ be a (closed) subspace of $\mathcal{H}$, and $U: \mathcal{H} \otimes \mathcal{K} \rightarrow \mathcal{H} \otimes \mathcal{P}$ and $\tilde{U}: \tilde{\mathcal{H}} \otimes \mathcal{K} \rightarrow$ $\tilde{\mathcal{H}} \otimes \mathcal{P}$ be two unitaries such that

$$
U\left(\tilde{h} \otimes \Omega^{\mathcal{K}}\right)=\tilde{U}\left(\tilde{h} \otimes \Omega^{\mathcal{K}}\right) \text { for all } \tilde{h} \in \tilde{\mathcal{H}} .
$$

We fix $\left\{\epsilon_{1}, \ldots, \epsilon_{d}\right\}$ to be an orthonormal basis of $\mathcal{P}$. The equation (1.2) is the analog of the equation (1.1) for our model and thus our model can be used for the setting where a quantum system interacts with a stream of copies of another quantum system in such a way that there is no backaction (so we get a Markovian type of dynamics) and such that there is a certain kind of subprocess. In the model of [9] the vacuum state $\Omega^{\mathcal{H}}$ of an atom plays an important role. For a model describing interaction of a quantum system with a stream of copies of another quantum sytem we need that the computations do not involve any fixed unit vector $\Omega^{\mathcal{H}}$ and we are able to achieve this in our model by using a pair of unitaries. Instead of $\Omega^{\mathcal{H}}$ we now have a kind of subprocess, described by $\tilde{U}$, which can be treated on the same level as the full process, described by $U$.

The main condition imposed on the unitary $U: \mathcal{H} \otimes \mathcal{K} \rightarrow \mathcal{H} \otimes \mathcal{P}$ in order to get a generalized interaction model is that $U\left(\tilde{\mathcal{H}} \otimes \Omega^{\mathcal{K}}\right) \subset \tilde{\mathcal{H}} \otimes \mathcal{P}$ (cf. Proposition 3.1 of [10] for an interesting consequence of this assumption). We can then define $\tilde{U}$ restricted to $\tilde{\mathcal{H}} \otimes \Omega^{\mathcal{K}}$ as $U$ restricted to $\tilde{\mathcal{H}} \otimes \Omega^{\mathcal{K}}$, and assume that $\mathcal{H} \otimes \mathcal{P}$ is big enough to allow a unitary extension $\tilde{U}: \tilde{\mathcal{H}} \otimes \mathcal{K} \rightarrow \tilde{\mathcal{H}} \otimes \mathcal{P}$. 
The focus of the study done here, as also in [9], is to bring out that certain multi-analytic operators of the multivariate operator theory are associated to noncommutative Markov chains and related models, and that these operators can be exploited as powerful tools. These operators occur as central objects in various context such as in the systems theory related works (cf. [3]) and noncommutative multivariable operator theory related works (cf. [14], [15]).

A tuple $\underline{T}=\left(T_{1}, \ldots, T_{d}\right)$ of operators $T_{i}$ 's on a common Hilbert space $\mathcal{L}$ is called a row contraction if $\sum_{i=1}^{d} T_{i} T_{i}^{*} \leq I$. In particular if $\sum_{i=1}^{d} T_{i} T_{i}^{*}=I$, then the tuple $\underline{T}=\left(T_{1}, \ldots, T_{d}\right)$ is called coisometric. We introduce the notation $\tilde{\Lambda}$ for the free semigroup with generators $1, \ldots, d$. Suppose $T_{1}, \ldots, T_{d} \in$ $\mathcal{B}(\mathcal{L})$ for a Hilbert space $\mathcal{L}$. If $\alpha \in \tilde{\Lambda}$ is the word $\alpha_{1} \ldots \alpha_{n}$ with length $|\alpha|=n$, where each $\alpha_{j} \in\{1, \ldots, d\}$, then $T_{\alpha}$ denote $T_{\alpha_{1}} \ldots T_{\alpha_{n}}$. For the empty word $\emptyset$ we define $|\emptyset|=0$ and $T_{\emptyset}=I$.

The unitary $U: \mathcal{H} \otimes \mathcal{K} \rightarrow \mathcal{H} \otimes \mathcal{P}$ from our model can be decomposed as

$$
U\left(h \otimes \Omega^{\mathcal{K}}\right)=\sum_{j=1}^{d} E_{j}^{*} h \otimes \epsilon_{j} \text { for } h \in \mathcal{H},
$$

where $E_{j}$ 's are some operators in $\mathcal{B}(\mathcal{H})$, for $j=1, \ldots, d$. Likewise there exist some operators $C_{j}$ 's in $\mathcal{B}(\tilde{\mathcal{H}})$ such that

$$
\tilde{U}\left(\tilde{h} \otimes \Omega^{\mathcal{K}}\right)=\sum_{j=1}^{d} C_{j}^{*} \tilde{h} \otimes \epsilon_{j} \text { for } \tilde{h} \in \tilde{\mathcal{H}} .
$$

Observe that $\sum_{j=1}^{d} E_{j} E_{j}^{*}=I$ and $\sum_{j=1}^{d} C_{j} C_{j}^{*}=I$, i.e., $\underline{E}$ and $\underline{C}$ are coisometric tuples. By equation (1.2)

$$
E_{j}^{*} \tilde{h}=C_{j}^{*} \tilde{h} \text { for all } \tilde{h} \in \tilde{\mathcal{H}}, j=1, \ldots, d .
$$

We recall from [5] that such tuple $\underline{E}=\left(E_{1}, \ldots, E_{d}\right)$ is called a lifting of $\underline{C}=\left(C_{1}, \ldots, C_{d}\right)$.

From a physicist perspective our model is a Markovian approximation of the repeated interaction between a quantum system and a stream of copies of another quantum system in such a way that there is no backaction. The change of an observable $X \in \mathcal{B}(\mathcal{H})$ until time $n$, compressed to $\mathcal{H}$, is written as

$$
Z_{n}(X):=\left.P_{\mathcal{H}} U(n)^{*}(X \otimes I) U(n)\right|_{\mathcal{H}} .
$$

From equation (1.3) it follows that $Z_{n}(X)=Z^{n}(X)$ where $Z(X)=$ $\sum_{i=1}^{d} E_{i} X E_{i}^{*}: B(\mathcal{H}) \rightarrow B(\mathcal{H})$ and $Z$ is called the transition operator of the noncommutative Markov chain.

In section 2 we develop our generalized repeated interaction model and obtain a coisometric operator which intertwines between the minimal isometric dilations of $\underline{E}$ and $\underline{C}$, and which will be crucial for the further investigation in this article. Using this an outgoing Cuntz scattering system in the sense of 3 is constructed for our model in section 3. Popescu introduced the minimal isometric dilation in [13] and the characteristic function in [14] of a row 
contraction, and systematically developed an extensive theory of row contractions (cf. [16], 17]). We use some of the concepts from Popescu's theory in this work.

For the outgoing Cuntz scattering system in section 4 we give a $\tilde{\Lambda}$-linear system with an input-output formalism. A multi-analytic operator appears here as the transfer function and in the next section we show that this transfer function can be derived from the intertwining coisometry of section 2 . In the scattering interpretation of the transfer function this now mediates between two processes. This together with a nice product formula obtained in Proposition 2.1 tells us that this identification of transfer function is a reminiscent of the scattering operator construction using wave operators in Lax-Phillips scattering theory [12, equation (1.5) (cf. [18), with one of the processes moving forward combined with the other moving backward. In [20] and [7] there are other approaches to transfer functions. Several works on transfer functions and on quantum systems using linear system theory can be found in recent theoretical physics and control theory surveys.

In section 5 we investigate in regard to our model what the notion of observability implies for the scattering theory and the theory of liftings. Some techniques used here are similar to those of scattering theory of noncommutative Markov chains introduced in 11. Characteristic functions for liftings, introduced in [5], are multi-analytic operators which classify certain class of liftings. Our model generalizes the setting of [9, and a comparison is done in section 6 between the transfer function of our model and the characteristic function for the associated lifting using the series expansion of the transfer function obtained in section 4 . As a consequence mathematically generalized interaction models get firmly linked into the theory of functional models.

\section{A Generalised Repeated Interaction Model}

We begin with three Hilbert spaces $\mathcal{H}, \mathcal{K}$ and $\mathcal{P}$ with unit vectors $\Omega^{\mathcal{K}} \in \mathcal{K}$ and $\Omega^{\mathcal{P}} \in \mathcal{P}$, and unitaries $U$ and $\tilde{U}$ as in equation (1.2). In $\mathcal{K}_{\infty}=\bigotimes_{i=1}^{\infty} \mathcal{K}$ and $\mathcal{P}_{\infty}=\bigotimes_{i=1}^{\infty} \mathcal{P}$ define $\Omega_{\infty}^{\mathcal{K}}:=\bigotimes_{i=1}^{\infty} \Omega^{\mathcal{K}}$ and $\Omega_{\infty}^{\mathcal{P}}:=\bigotimes_{i=1}^{\infty} \Omega^{\mathcal{P}}$ respectively. We denote $m$-th copy of $\Omega^{\mathcal{K}}$ in $\Omega_{\infty}^{\mathcal{K}}$ by $\Omega_{m}^{\mathcal{K}}$ and in terms of this we introduce the notation $\Omega_{[m, n]}^{\mathcal{K}}:=\Omega_{m}^{\mathcal{K}} \otimes \cdots \otimes \Omega_{n}^{\mathcal{K}}$. Identify $\mathcal{K}_{[m, n]}$ with $\Omega_{[1, m-1]}^{\mathcal{K}} \otimes \mathcal{K}_{[m, n]} \otimes$ $\Omega_{[n+1, \infty)}^{\mathcal{K}}, \mathcal{H}$ with $\mathcal{H} \otimes \Omega_{\infty}^{\mathcal{K}}$ as a subspace of $\mathcal{H} \otimes \mathcal{K}_{\infty}$ and $\tilde{\mathcal{H}}$ with $\tilde{\mathcal{H}} \otimes \Omega_{\infty}^{\mathcal{K}}$ as a subspace of $\tilde{\mathcal{H}} \otimes \mathcal{K}_{\infty}$. Similar notations with respect to $\mathcal{P}$ are also used. For simplicity we assume that $d$ is finite but all the results here can be derived also for $d=\infty$.

Associate a row contraction $\underline{E}$ to the unitary $U$ as in equation (1.3) and define isometries

$$
\widehat{V}_{j}^{E}(h \otimes \eta):=U^{*}\left(h \otimes \epsilon_{j}\right) \otimes \eta \text { for } j=1, \ldots, d,
$$

on the elementary tensors $h \otimes \eta \in \mathcal{H} \otimes \mathcal{K}_{\infty}$ and extend it linearly to obtain $\widehat{V}_{j}^{E} \in \mathcal{B}\left(\mathcal{H} \otimes \mathcal{K}_{\infty}\right)$ for $j=1, \ldots, d$. We recall that a lifting $\underline{T}=\left(T_{1}, \ldots, T_{d}\right)$ of any row contraction $\underline{S}=\left(S_{1}, \ldots, S_{d}\right)$ is called its isometric dilation if 
$T_{i}$ 's are isometries with orthogonal ranges. It can be easily verified that $\widehat{\widehat{V}}^{E}=\left(\widehat{V}_{1}^{E}, \ldots, \widehat{V}_{d}^{E}\right)$ on the space $\mathcal{H} \otimes \mathcal{K}_{\infty}$ is an isometric dilation of $\underline{E}=$ $\left(E_{1}, \ldots, E_{d}\right)$. If $h \in \mathcal{H}$ and $k_{1} \in \mathcal{K}$, then there exist $h_{i} \in \mathcal{H}$ for $i=1, \ldots, d$ such that $U^{*}\left(\sum_{i=1}^{d} h_{i} \otimes \epsilon_{i}\right)=h \otimes k_{1}$ because $U$ is a unitary. This implies

$$
\sum_{i=1}^{d} \widehat{V}_{i}^{E}\left(h_{i} \otimes \Omega_{\infty}^{\mathcal{K}}\right)=h \otimes k_{1} \otimes \Omega_{[2, \infty)}^{\mathcal{K}}
$$

In addition if $k_{2} \in \mathcal{K}$, then

$\sum_{i=1}^{d} \widehat{V}_{i}^{E}\left(h_{i} \otimes k_{2} \otimes \Omega_{[2, \infty)}^{\mathcal{K}}\right)=U^{*}\left(\sum_{i=1}^{d} h_{i} \otimes \epsilon_{i}\right) \otimes k_{2} \otimes \Omega_{[3, \infty)}^{\mathcal{K}}=h \otimes k_{1} \otimes k_{2} \otimes \Omega_{[3, \infty)}^{\mathcal{K}}$.

By induction we conclude that

$$
\mathcal{H} \otimes \mathcal{K}_{\infty}=\overline{\operatorname{span}}\left\{\widehat{V}_{\alpha}^{E}\left(h \otimes \Omega_{\infty}^{\mathcal{K}}\right): h \in \mathcal{H}, \alpha \in \tilde{\Lambda}\right\},
$$

i.e., $\widehat{V}^{E}$ is the minimal isometric dilation of $\underline{E}$. Note that the minimal isometric dilation is unique up to unitary equivalence (cf. [13]).

Similarly, associate a row contraction $\underline{C}$ to the unitary $\tilde{U}$ as in equation (1.4) and define isometries

$$
\widehat{V}_{j}^{C}(\tilde{h} \otimes \eta):=\tilde{U}^{*}\left(\tilde{h} \otimes \epsilon_{j}\right) \otimes \eta \text { for } j=1, \ldots, d
$$

on the elementary tensors $\tilde{h} \otimes \eta \in \tilde{\mathcal{H}} \otimes \mathcal{K}_{\infty}$ and extend it linearly to obtain $\widehat{V}_{j}^{C} \in \mathcal{B}\left(\tilde{\mathcal{H}} \otimes \mathcal{K}_{\infty}\right)$ for $j=1, \ldots, d$. The tuple $\widehat{V}^{C}=\left(\widehat{V}_{1}^{C}, \ldots, \widehat{V}_{d}^{C}\right)$ on the space $\tilde{\mathcal{H}} \otimes \mathcal{K}_{\infty}$ is the minimal isometric dilation of $\underline{C}=\left(C_{1}, \ldots, C_{d}\right)$. Recall that

$$
U_{m}: \mathcal{H} \otimes \mathcal{K}_{\infty} \rightarrow \mathcal{H} \otimes \mathcal{K}_{[1, m-1]} \otimes \mathcal{P}_{m} \otimes \mathcal{K}_{[m+1, \infty)}
$$

is nothing but the operator which acts as $U$ on $\mathcal{H} \otimes \mathcal{K}_{m}$ and fixes other factors of the infinite tensor products. Similarly, we define $\tilde{U}_{m}$ using $\tilde{U}$.

Proposition 2.1. Let $P_{n}:=P_{\tilde{\mathcal{H}}} \otimes I_{\mathcal{P}_{[1, n]}} \otimes I_{\mathcal{K}_{[n+1, \infty)}} \in \mathcal{B}\left(\mathcal{H} \otimes \mathcal{P}_{[1, n]} \otimes \mathcal{K}_{[n+1, \infty)}\right)$ for $n \in \mathbb{N}$. Then

$$
s o t-\lim _{n \rightarrow \infty} \tilde{U}_{1}^{*} \ldots \tilde{U}_{n}^{*} P_{n} U_{n} \ldots U_{1}
$$

exists and this limit defines a coisometry $\widehat{W}: \mathcal{H} \otimes \mathcal{K}_{\infty} \rightarrow \tilde{\mathcal{H}} \otimes \mathcal{K}_{\infty}$. Its adjoint $\widehat{W}^{*}: \tilde{\mathcal{H}} \otimes \mathcal{K}_{\infty} \rightarrow \mathcal{H} \otimes \mathcal{K}_{\infty}$ is given by

$$
\widehat{W}^{*}=\operatorname{sot}-\lim _{n \rightarrow \infty} U_{1}^{*} \ldots U_{n}^{*} \tilde{U}_{n} \ldots \tilde{U}_{1} .
$$

Here sot stands for the strong operator topology.

Proof. At first we construct the adjoint $\widehat{W}^{*}$. For that consider the dense subset $\bigcup_{m \geq 1} \tilde{\mathcal{H}} \otimes \mathcal{K}_{[1, m]}$ of $\tilde{\mathcal{H}} \otimes \mathcal{K}_{\infty}$ and let an arbitrary simple tensor element of this dense subset be $\tilde{h} \otimes k_{1} \otimes \ldots \otimes k_{\ell} \otimes \Omega_{[\ell+1, \infty)}^{\mathcal{K}}$ for some $\ell \in \mathbb{N}, \tilde{h} \in \tilde{\mathcal{H}}$ and $k_{i} \in \mathcal{K}_{i}$. Set $a_{p}=U_{1}^{*} \ldots U_{p}^{*} \tilde{U}_{p} \ldots \tilde{U}_{1}\left(\tilde{h} \otimes k_{1} \otimes \ldots \otimes k_{\ell} \otimes \Omega_{[\ell+1, \infty)}^{\mathcal{K}}\right)$ for $p \in \mathbb{N}$. 
Since $U\left(\tilde{h} \otimes \Omega^{\mathcal{K}}\right)=\tilde{U}\left(\tilde{h} \otimes \Omega^{\mathcal{K}}\right)$ for all $\tilde{h} \in \tilde{\mathcal{H}}$, we have $a_{\ell}=a_{\ell+n}$ for all $n \in \mathbb{N}$. Therefore we deduce that

$$
\lim _{n \rightarrow \infty} U_{1}^{*} \ldots U_{n}^{*} \tilde{U}_{n} \ldots \tilde{U}_{1}\left(\tilde{h} \otimes k_{1} \otimes \ldots \otimes k_{\ell} \otimes \Omega_{[\ell+1, \infty)}^{\mathcal{K}}\right)
$$

exists. Because $U$ and $\tilde{U}$ are unitaries, we obtain an isometric extension $\widehat{W}^{*}$ to the whole of $\tilde{\mathcal{H}} \otimes \mathcal{K}_{\infty}$. Thus its adjoint is a coisometry $\widehat{W}: \mathcal{H} \otimes \mathcal{K}{ }_{\infty} \rightarrow \tilde{\mathcal{H}} \otimes \mathcal{K}_{\infty}$.

Now we will derive the limit form for $\widehat{W}$ as claimed in the statement of the proposition. If $h \otimes \eta \in \mathcal{H} \otimes \mathcal{K}_{[1, k]}, \tilde{h} \otimes \tilde{\eta} \in \tilde{\mathcal{H}} \otimes \mathcal{K}_{[1, n]}$ and $k \leq n$, then

$$
\begin{aligned}
\langle\widehat{W}(h \otimes \eta), \tilde{h} \otimes \tilde{\eta}\rangle & =\left\langle h \otimes \tilde{\eta}, \widehat{W}^{*}(\tilde{h} \otimes \tilde{\eta})\right\rangle \\
& =\left\langle h \otimes \eta, U_{1}^{*} \ldots U_{n}^{*} \tilde{U}_{n} \ldots \tilde{U}_{1}(\tilde{h} \otimes \tilde{\eta})\right\rangle \\
& =\left\langle\tilde{U}_{1}^{*} \ldots \tilde{U}_{n}^{*} P_{n} U_{n} \ldots U_{1}(h \otimes \eta), \tilde{h} \otimes \tilde{\eta}\right\rangle .
\end{aligned}
$$

Consequently $\widehat{W}=\operatorname{sot}-\lim _{n \rightarrow \infty} \tilde{U}_{1}^{*} \ldots \tilde{U}_{n}^{*} P_{n} U_{n} \ldots U_{1}$ on a dense subset and therefore it can be extended to the whole of $\mathcal{H} \otimes \mathcal{K}_{\infty}$.

Observe that

$$
\widehat{W}^{*}\left(\tilde{h} \otimes \Omega_{\infty}^{\mathcal{K}}\right)=\tilde{h} \otimes \Omega_{\infty}^{\mathcal{K}} \text { for all } \tilde{h} \in \tilde{\mathcal{H}}
$$

Next we show that this coisometry $\widehat{W}$ intertwines between $\widehat{V}_{j}^{E}$ and $\widehat{V}_{j}^{C}$ for all $j=1, \ldots, d$. For $j=1, \ldots, d$, define

$$
\begin{aligned}
S_{j}: \mathcal{H} \otimes \mathcal{K}_{\infty} & \rightarrow \mathcal{H} \otimes \mathcal{P}_{1} \otimes \mathcal{K}_{[2, \infty)} \\
h \otimes \eta & \mapsto h \otimes \epsilon_{j} \otimes \eta
\end{aligned}
$$

The following are immediate:

(1) $S_{j}^{*}\left(h \otimes p_{1} \otimes \eta\right)=\left\langle\epsilon_{j}, p_{1}\right\rangle(h \otimes \eta)$ for $\left(h \otimes p_{1} \otimes \eta\right) \in \mathcal{H} \otimes \mathcal{P}_{1} \otimes \mathcal{K}_{[2, \infty)}$.

(2) $\widehat{V}_{j}^{E}(h \otimes \eta)=U_{1}^{*} S_{j}(h \otimes \eta)$ for $h \otimes \eta \in \mathcal{H} \otimes \mathcal{K}_{\infty}$.

(3) $\widehat{V}_{j}^{C}(\tilde{h} \otimes \eta)=\tilde{U}_{1}^{*} S_{j}(\tilde{h} \otimes \eta)$ for $\tilde{h} \otimes \eta \in \tilde{\mathcal{H}} \otimes \mathcal{K}_{\infty}$.

Proposition 2.2. If $\widehat{W}$ is as in Proposition 2.1, then

$$
\widehat{W} \widehat{V}_{j}^{E}=\widehat{V}_{j}^{C} \widehat{W}, \quad \widehat{V}_{j}^{E} \widehat{W}^{*}=\widehat{W}^{*} \widehat{V}_{j}^{C} \text { for all } j=1, \ldots, d \text {. }
$$

Proof. If $h \in \mathcal{H}, \eta \in \mathcal{K}_{\infty}, \tilde{h} \in \tilde{\mathcal{H}}$ and $k_{i} \in \mathcal{K}_{i}$, then by the three observations that were noted preceding this proposition we obtain for $j=1, \ldots, d$

$$
\begin{aligned}
& \left\langle\widehat{W} \widehat{V}_{j}^{E}(h \otimes \eta), \tilde{h} \otimes k_{1} \otimes \ldots \otimes k_{\ell} \otimes \Omega_{[\ell+1, \infty)}^{\mathcal{K}}\right\rangle \\
= & \left\langle U^{*}\left(h \otimes \epsilon_{j}\right) \otimes \eta, U_{1}^{*} \ldots U_{\ell}^{*} \tilde{U}_{\ell} \ldots \tilde{U}_{1}\left(\tilde{h} \otimes k_{1} \otimes \ldots \otimes k_{\ell} \otimes \Omega_{[\ell+1, \infty)}^{\mathcal{K}}\right)\right\rangle .
\end{aligned}
$$


Substituting $\tilde{U}\left(\tilde{h} \otimes k_{1}\right)=\sum_{i} \tilde{h}^{(i)} \otimes k_{1}^{(i)}$ where $\tilde{h}^{(i)} \in \tilde{\mathcal{H}}$ and $k_{1}^{(i)} \in \mathcal{K}$ we obtain

$$
\begin{aligned}
& \left\langle\widehat{W} \widehat{V}_{j}^{E}(h \otimes \eta), \tilde{h} \otimes k_{1} \otimes \ldots \otimes k_{\ell} \otimes \Omega_{[\ell+1, \infty)}^{\mathcal{K}}\right\rangle \\
= & \left\langle h \otimes \epsilon_{j} \otimes \eta, U_{2}^{*} \ldots U_{\ell}^{*} \tilde{U}_{\ell} \ldots \tilde{U}_{2}\left(\sum_{i}\left(\tilde{h}^{(i)} \otimes k_{1}^{(i)}\right)\right.\right. \\
& \left.\left.\otimes k_{2} \otimes \ldots \otimes k_{\ell} \otimes \Omega_{[\ell+1, \infty)}^{\mathcal{K}}\right)\right\rangle \\
= & \sum_{i}\left\langle\epsilon_{j}, k_{1}^{(i)}\right\rangle\left\langle h \otimes \eta, \widehat{W}^{*}\left(\tilde{h}^{(i)} \otimes k_{2} \otimes \ldots \otimes k_{\ell} \otimes \Omega_{[\ell+1, \infty)}^{\mathcal{K}}\right)\right\rangle \\
= & \left\langle\widehat{W}(h \otimes \eta), S_{j}^{*} \tilde{U}_{1}\left(\tilde{h} \otimes k_{1} \otimes \ldots \otimes k_{\ell} \otimes \Omega_{[\ell+1, \infty)}^{\mathcal{K}}\right)\right\rangle \\
= & \left\langle\tilde{U}_{1}^{*} S_{j} \widehat{W}\left((h \otimes \eta), \tilde{h} \otimes k_{1} \otimes \ldots \otimes k_{\ell} \otimes \Omega_{[\ell+1, \infty)}^{\mathcal{K}}\right\rangle\right. \\
= & \left\langle\widehat{V}_{j}^{C} \widehat{W}(h \otimes \eta), \tilde{h} \otimes k_{1} \otimes \ldots \otimes k_{\ell} \otimes \Omega_{[\ell+1, \infty)}^{\mathcal{K}}\right\rangle .
\end{aligned}
$$

Hence $\widehat{W} \widehat{V}_{j}^{E}=\widehat{V}_{j}^{C} \widehat{W}$ for all $j=1, \ldots, d$. To obtain the other equation of the proposition we again use the last two of the three observations as follows: For $j=1, \ldots, d$

$$
\begin{aligned}
& \widehat{W}^{*} \widehat{V}_{j}^{C}\left(\tilde{h} \otimes k_{1} \otimes \ldots \otimes k_{\ell} \otimes \Omega_{[\ell+1, \infty)}^{\mathcal{K}}\right) \\
= & \widehat{W}^{*} \tilde{U}_{1}^{*}\left(\tilde{h} \otimes \epsilon_{j} \otimes k_{1} \otimes \ldots \otimes k_{\ell} \otimes \Omega_{[\ell+2, \infty)}^{\mathcal{K}}\right) \\
= & U_{1}^{*} U_{2}^{*} \ldots U_{\ell+1}^{*} \tilde{U}_{\ell+1} \ldots \tilde{U}_{2} \tilde{U}_{1} \tilde{U}_{1}^{*}\left(\tilde{h} \otimes \epsilon_{j} \otimes k_{1} \otimes \ldots \otimes k_{\ell} \otimes \Omega_{[\ell+2, \infty)}^{\mathcal{K}}\right) \\
= & U_{1}^{*} U_{2}^{*} \ldots U_{\ell+1}^{*} \tilde{U}_{\ell+1} \ldots \tilde{U}_{2} S_{j}\left(\tilde{h} \otimes k_{1} \otimes \ldots \otimes k_{\ell} \otimes \Omega_{[\ell+1, \infty)}^{\mathcal{K}}\right) \\
= & U_{1}^{*} S_{j} U_{1}^{*} \ldots U_{\ell}^{*} \tilde{U}_{\ell} \ldots \tilde{U}_{1}\left(\tilde{h} \otimes k_{1} \otimes \ldots \otimes k_{\ell} \otimes \Omega_{[\ell+1, \infty)}^{\mathcal{K}}\right) \\
= & \widehat{V}_{j}^{E} \widehat{W}^{*}\left(\tilde{h} \otimes k_{1} \otimes \ldots \otimes k_{\ell} \otimes \Omega_{[\ell+1, \infty)}^{\mathcal{K}}\right)
\end{aligned}
$$

Further define

$$
\begin{gathered}
\left(\mathcal{H} \otimes \mathcal{K}_{\infty}\right)^{\circ}:=\left(\mathcal{H} \otimes \mathcal{K}_{\infty}\right) \ominus\left(\tilde{\mathcal{H}} \otimes \Omega_{\infty}^{\mathcal{K}}\right), \\
\left(\tilde{\mathcal{H}} \otimes \mathcal{K}_{\infty}\right)^{\circ}:=\left(\tilde{\mathcal{H}} \otimes \mathcal{K}_{\infty}\right) \ominus\left(\tilde{\mathcal{H}} \otimes \Omega_{\infty}^{\mathcal{K}}\right) \text { and } \mathcal{H}^{\circ}:=\mathcal{H} \ominus \tilde{\mathcal{H}} .
\end{gathered}
$$

Let $\sum_{i=1}^{k} \xi_{i} \otimes \eta_{i} \in\left(\mathcal{H} \otimes \mathcal{K}_{\infty}\right)^{\circ}$ and $\tilde{h} \in \tilde{\mathcal{H}}$. Then for $j=1, \ldots, d$

$$
\begin{aligned}
\left\langle\widehat{V}_{j}^{E}\left(\sum_{i} \xi_{i} \otimes \eta_{i}\right), \tilde{h} \otimes \Omega_{\infty}^{\mathcal{K}}\right\rangle & =\left\langle\sum_{i} U^{*}\left(\xi_{i} \otimes \epsilon_{j}\right) \otimes \eta_{i}, \tilde{h} \otimes \Omega_{\infty}^{\mathcal{K}}\right\rangle \\
& =\left\langle\sum_{i} \xi_{i} \otimes \epsilon_{j} \otimes \eta_{i}, \tilde{U}\left(\tilde{h} \otimes \Omega_{1}^{\mathcal{K}}\right) \otimes \Omega_{[2, \infty)}^{\mathcal{K}}\right\rangle=0
\end{aligned}
$$

because $\tilde{U}$ maps into $\tilde{\mathcal{H}} \otimes \mathcal{P}$ and $\sum_{i=1}^{k} \xi_{i} \otimes \eta_{i} \perp \tilde{\mathcal{H}} \otimes \Omega^{\mathcal{K}}$. Therefore $\widehat{V}_{j}^{E}(\mathcal{H} \otimes$ $\left.\mathcal{K}_{\infty}\right)^{\circ} \subset\left(\mathcal{H} \otimes \mathcal{K}_{\infty}\right)^{\circ}$ for $j=1, \ldots, d$. Similarly $\widehat{V}_{j}^{C}\left(\tilde{\mathcal{H}} \otimes \mathcal{K}_{\infty}\right)^{\circ} \subset\left(\tilde{\mathcal{H}} \otimes \mathcal{K}_{\infty}\right)^{\circ}$ for $j=1, \ldots, d$. Set $V_{j}^{E}:=\left.\widehat{V}_{j}^{E}\right|_{\left(\mathcal{H} \otimes \mathcal{K}_{\infty}\right)^{\circ}}$ and $V_{j}^{C}:=\left.\widehat{V}_{j}^{C}\right|_{\left(\tilde{\mathcal{H}} \otimes \mathcal{K}_{\infty}\right)}$ for $j=$ $1, \ldots, d$. If we define

$$
W^{*}:=\left.\widehat{W}^{*}\right|_{\left(\tilde{\mathcal{H}} \otimes \mathcal{K}_{\infty}\right)^{\circ}},
$$


then by equation $(2.2)$ it follows that $W^{*} \in \mathcal{B}\left(\left(\tilde{\mathcal{H}} \otimes \mathcal{K}_{\infty}\right)^{\circ},\left(\mathcal{H} \otimes \mathcal{K}_{\infty}\right)^{\circ}\right)$. The operator $W^{*}$ is an isometry because it is a restriction of an isometry and $W$, the adjoint of $W^{*}$, is the restriction of $\widehat{W}$ to $\left(\mathcal{H} \otimes \mathcal{K}_{\infty}\right)^{\circ}$, i.e., $W=\left.\widehat{W}\right|_{\left(\mathcal{H} \otimes \mathcal{K}_{\infty}\right)^{\circ}}$.

Remark 2.3. It follows that

$$
W V_{j}^{E}=V_{j}^{C} W
$$

for $j=1, \ldots, d$.

\section{Outgoing Cuntz Scattering Systems}

In this section we aim to construct an outgoing Cuntz scattering system (cf. 3 ) for our model. This will assist us in the next section to work with an input-output formalism and to associate a transfer function to the model.

Following are some notions from the multivariable operator theory:

Definition 3.1. Suppose $\underline{T}=\left(T_{1}, \ldots, T_{d}\right)$ is a row contraction where $T_{i} \in$ $\mathcal{B}(\mathcal{L})$.

(1) If $T_{i}$ 's are isometries with orthogonal ranges, then the tuple $\underline{T}=\left(T_{1}, \ldots\right.$, $\left.T_{d}\right)$ is called a row isometry.

(2) If $\overline{\operatorname{span}}_{j=1, \ldots, d} T_{j} \mathcal{L}=\mathcal{L}$ and $\underline{T}=\left(T_{1}, \ldots, T_{d}\right)$ is a row isometry, then $\underline{T}$ is called a row unitary.

(3) If there exist a subspace $\mathcal{E}$ of $\mathcal{L}$ such that $\mathcal{L}=\bigoplus_{\alpha \in \tilde{\Lambda}} T_{\alpha} \mathcal{E}$ and $\underline{T}=$ $\left(T_{1}, \ldots, T_{d}\right)$ is a row isometry, then $\underline{T}$ is called a row shift and $\mathcal{E}$ is called a wandering subspace of $\mathcal{L}$ w.r.t. $\underline{T}$.

Definition 3.2. A collection $\left(\mathcal{L}, \underline{V}=\left(V_{1}, \ldots, V_{d}\right), \mathcal{G}_{*}^{+}, \mathcal{G}\right)$ is called an outgoing Cuntz scattering system (cf. [3]), if $\underline{V}$ is a row isometry on the Hilbert space $\mathcal{L}$, and $\mathcal{G}_{*}^{+}$and $\mathcal{G}$ are subspaces of $\mathcal{L}$ such that

(1) for $\mathcal{E}_{*}:=\mathcal{L} \ominus \overline{\operatorname{span}}_{j=1, \ldots, d} V_{j} \mathcal{L}$, the tuple $\underline{V} \mid \mathcal{G}_{*}^{+}$is a row shift where $\mathcal{G}_{*}^{+}=\bigoplus_{\alpha \in \tilde{\Lambda}} V_{\alpha} \mathcal{E}_{*}$.

(2) there exist $\mathcal{E}:=\mathcal{G} \ominus \overline{\operatorname{span}}_{j=1, \ldots, d} V_{j} \mathcal{G}$ with $\mathcal{G}=\bigoplus_{\alpha \in \tilde{\Lambda}} V_{\alpha} \mathcal{E}$, i.e., $\left.\underline{V}\right|_{\mathcal{G}}$ is a row shift.

In the above definition the part (1) is the Wold decomposition (cf. [13]) of the row isometry $\underline{V}$ and therefore $\mathcal{G}_{*}^{+}$can be derived from $\underline{V}$. But $\mathcal{G}_{*}^{+}$is included in the data because it helps in describing the scattering phenomenon. We continue using the notations from the previous section. $\widehat{V}_{j}^{E}$ 's are isometries with orthogonal ranges and because $\left(\epsilon_{j}\right)_{j=1}^{d}$ is an orthonormal basis of $\mathcal{P}$, we have

$$
\overline{\operatorname{span}}_{j=1, \ldots, d} \widehat{V}_{j}^{E}\left(\mathcal{H} \otimes \mathcal{K}_{\infty}\right)=\mathcal{H} \otimes \mathcal{K}_{\infty}
$$

Thus $\widehat{V}^{E}$ is a row unitary on $\mathcal{H} \otimes \mathcal{K}_{\infty}$. Now using the fact that $V_{j}^{E}=$ $\left.\widehat{V}_{j}^{E}\right|_{\left(\mathcal{H} \otimes \mathcal{K}_{\infty}\right)}$ ' we infer that $V_{j}^{E}$ 's are isometries with orthogonal ranges. Therefore $\underline{V}^{E}$ is a row isometry on $\left(\mathcal{H} \otimes \mathcal{K}_{\infty}\right)^{\circ}$. 
Proposition 3.3. If $\mathcal{Y}:=\tilde{\mathcal{H}} \otimes\left(\Omega_{1}^{\mathcal{K}}\right)^{\perp} \otimes \Omega_{[2, \infty)}^{\mathcal{K}} \subset \tilde{\mathcal{H}} \otimes \mathcal{K}_{\infty}$, then

$$
W^{*} \mathcal{Y} \perp \overline{\operatorname{span}}_{j=1, \ldots, d} V_{j}^{E}\left(\mathcal{H} \otimes \mathcal{K}_{\infty}\right)^{\circ} .
$$

Proof. By Proposition 2.1 it is easy to see that

$$
W^{*} \mathcal{Y}=U_{1}^{*} \tilde{U}_{1} \mathcal{Y} \subset \mathcal{H} \otimes \mathcal{K}_{1} \otimes \Omega_{[2, \infty)}^{\mathcal{K}} .
$$

Let $\tilde{h}_{i} \in \tilde{\mathcal{H}}$ and $k_{i} \perp \Omega_{1}^{\mathcal{K}}$ for $i=1, \ldots, n$, i.e., $\sum_{i} \tilde{h}_{i} \otimes k_{i} \otimes \Omega_{[2, \infty)}^{\mathcal{K}} \in \mathcal{Y}$. For $\sum_{k} h_{k} \otimes \eta_{k} \in\left(\mathcal{H} \otimes \mathcal{K}_{\infty}\right)^{\circ}$ with $h_{k} \in \mathcal{H}$ and $\eta_{k} \in \mathcal{K}_{\infty}$

$$
\begin{aligned}
& \left\langle W^{*}\left(\sum_{i} \tilde{h}_{i} \otimes k_{i} \otimes \Omega_{[2, \infty)}^{\mathcal{K}}\right), V_{j}^{E}\left(\sum_{k} h_{k} \otimes \eta_{k}\right)\right\rangle \\
= & \left\langle U^{*} \tilde{U}\left(\sum_{i} \tilde{h}_{i} \otimes k_{i}\right) \otimes \Omega_{[2, \infty)}^{\mathcal{K}}, \sum_{k} U^{*}\left(h_{k} \otimes \epsilon_{j}\right) \otimes \eta_{k}\right\rangle \\
= & \left\langle\tilde{U}\left(\sum_{i} \tilde{h}_{i} \otimes k_{i}\right) \otimes \Omega_{[2, \infty)}^{\mathcal{K}}, \sum_{k} h_{k} \otimes \epsilon_{j} \otimes \eta_{k}\right\rangle=0 .
\end{aligned}
$$

The last equality holds because $\sum_{k} h_{k} \otimes \eta_{k} \perp \tilde{\mathcal{H}} \otimes \Omega_{\infty}^{\mathcal{K}}$. Thus $W^{*} \mathcal{Y} \perp$ $\overline{\operatorname{span}}_{j=1, \ldots, d} V_{j}^{E}\left(\mathcal{H} \otimes \mathcal{K}_{\infty}\right)^{\circ}$.

The following Proposition gives an explicit description of the Wold decomposition of $\underline{V}^{E}$ :

Proposition 3.4. If $\mathcal{Y}$ is defined as in the previous proposition, then $W^{*} \mathcal{Y}$ is a wandering subspace of $\underline{V}^{E}$, i.e., $V_{\alpha}^{E}\left(W^{*} \mathcal{Y}\right) \perp V_{\beta}^{E}\left(W^{*} \mathcal{Y}\right)$ whenever $\alpha, \beta \in \tilde{\Lambda}$, $\alpha \neq \beta$, and

$$
W^{*} \mathcal{Y}=\left(\mathcal{H} \otimes \mathcal{K}_{\infty}\right)^{\circ} \ominus \overline{\operatorname{span}}_{j=1, \ldots, d} V_{j}^{E}\left(\mathcal{H} \otimes \mathcal{K}_{\infty}\right)^{\circ} .
$$

Proof. By Proposition 3.3 it is immediate that $V_{\alpha}^{E}\left(W^{*} \mathcal{Y}\right) \perp V_{\beta}^{E}\left(W^{*} \mathcal{Y}\right)$ whenever $\alpha, \beta \in \tilde{\Lambda}, \alpha \neq \beta$ and $W^{*} \mathcal{Y} \subset\left(\mathcal{H} \otimes \mathcal{K}_{\infty}\right)^{\circ} \ominus \overline{\operatorname{span}}_{j=1, \ldots, d} V_{j}^{E}\left(\mathcal{H} \otimes \mathcal{K}_{\infty}\right)^{\circ}$. The only thing that remains to be shown is that

$$
\left(\mathcal{H} \otimes \mathcal{K}_{\infty}\right)^{\circ} \ominus \overline{\operatorname{span}}_{j=1, \ldots, d} V_{j}^{E}\left(\mathcal{H} \otimes \mathcal{K}_{\infty}\right)^{\circ} \subset W^{*} \mathcal{Y} .
$$

Let $x \in\left(\mathcal{H} \otimes \mathcal{K}_{\infty}\right)^{\circ} \ominus \overline{\operatorname{span}}_{j=1, \ldots, d} V_{j}^{E}\left(\mathcal{H} \otimes \mathcal{K}_{\infty}\right)^{\circ}$. Write down the decomposition of $x$ as $x_{1} \oplus x_{2}$ w.r.t. $W^{*} \mathcal{Y} \oplus\left(W^{*} \mathcal{Y}\right)^{\perp}$. So $x-x_{1}=x_{2}$ is orthogonal to both $\overline{\operatorname{span}}_{j=1, \ldots, d} V_{j}^{E}\left(\mathcal{H} \otimes \mathcal{K}_{\infty}\right)^{\circ}$ and $W^{*} \mathcal{Y}$. Now we show that if any element in $\left(\mathcal{H} \otimes \mathcal{K}_{\infty}\right)^{\circ}$ is orthogonal to $\overline{\operatorname{span}}_{j=1, \ldots, d} V_{j}^{E}\left(\mathcal{H} \otimes \mathcal{K}_{\infty}\right)^{\circ}$ and $W^{*} \mathcal{Y}$, then it is the zero vector. Let $x_{0}$ be such an element. Because $x_{0} \in\left(\mathcal{H} \otimes \mathcal{K}_{\infty}\right)^{\circ}$ and $x_{0} \perp W^{*} \mathcal{Y}$,

$$
x_{0} \perp U^{*}\left(\tilde{\mathcal{H}} \otimes \epsilon_{j}\right) \otimes \Omega_{[2, \infty)}^{\mathcal{K}}
$$

for $j=1, \ldots, d$. This implies $x_{0} \perp \overline{\operatorname{span}}_{j=1, \ldots, d} \widehat{V}_{j}^{E}\left(\tilde{\mathcal{H}} \otimes \Omega_{\infty}^{\mathcal{K}}\right)$. We also know that

$$
x_{0} \perp \overline{\operatorname{span}}_{j=1, \ldots, d} V_{j}^{E}\left(\mathcal{H} \otimes \mathcal{K}_{\infty}\right)^{\circ}\left(=\overline{\operatorname{span}}_{j=1, \ldots, d} \widehat{V}_{j}^{E}\left(\mathcal{H} \otimes \mathcal{K}_{\infty}\right)^{\circ}\right) .
$$

Therefore

$$
x_{0} \perp \overline{\operatorname{span}}_{j=1, \ldots, d} \widehat{V}_{j}^{E}\left(\mathcal{H} \otimes \mathcal{K}_{\infty}\right) .
$$

Since $\widehat{V}^{E}$ is a row unitrary, $x_{0} \perp \mathcal{H} \otimes \mathcal{K}_{\infty}$. So $x_{0}=0$ and hence $x=x_{1} \in$ $W^{*} \mathcal{Y}$. We conclude that $\left(\mathcal{H} \otimes \mathcal{K}_{\infty}\right)^{\circ} \ominus \overline{\operatorname{span}}_{j=1, \ldots, d} V_{j}^{E}\left(\mathcal{H} \otimes \mathcal{K}_{\infty}\right)^{\circ} \subset W^{*} \mathcal{Y}$. 
Proposition 3.5. If $\mathcal{E}:=\mathcal{H} \otimes\left(\Omega_{1}^{\mathcal{K}}\right)^{\perp} \otimes \Omega_{[2, \infty)}^{\mathcal{K}} \subset\left(\mathcal{H} \otimes \mathcal{K}_{\infty}\right)^{\circ}$, then $V_{\alpha}^{E} \mathcal{E} \perp V_{\beta}^{E} \mathcal{E}$ whenever $\alpha, \beta \in \tilde{\Lambda}, \alpha \neq \beta$ and $\left(\mathcal{H} \otimes \mathcal{K}_{\infty}\right)^{\circ}=\mathcal{H}^{\circ} \oplus \bigoplus_{\alpha \in \tilde{\Lambda}} V_{\alpha}^{E} \mathcal{E}$.

Proof. If $|\alpha|=|\beta|$ and $\alpha \neq \beta$, then it is easy to see that $V_{\alpha}^{E} \mathcal{E} \perp V_{\beta}^{E} \mathcal{E}$ because ranges of $V_{j}^{E}$ 's are mutually orthogonal. If $|\alpha| \neq|\beta|$ (without loss of generality we can assume that $|\alpha|>|\beta|$ ), then by taking the inner product at the tensor factor $\mathcal{K}_{|\alpha|+1}$ we obtain $V_{\alpha}^{E} \mathcal{E} \perp V_{\beta}^{E} \mathcal{E}$.

To prove the second part of the proposition, observe that for $n \in \mathbb{N}$,

$$
\begin{aligned}
& \mathcal{H} \otimes \mathcal{K}_{[1, n]} \otimes \Omega_{[n+1, \infty]}^{\mathcal{K}} \\
= & \left(\mathcal{H} \otimes \Omega_{\infty}^{\mathcal{K}}\right) \oplus\left(\mathcal{H} \otimes\left(\Omega_{1}^{\mathcal{K}}\right)^{\perp} \otimes \Omega_{[2, \infty)}^{\mathcal{K}}\right) \oplus\left(\mathcal{H} \otimes \mathcal{K}_{1} \otimes\right. \\
& \left.\left(\Omega_{2}^{\mathcal{K}}\right)^{\perp} \otimes \Omega_{[3, \infty)}^{\mathcal{K}}\right) \oplus \cdots \oplus\left(\mathcal{H} \otimes \mathcal{K}_{[1, n-1]} \otimes\left(\Omega_{n}^{\mathcal{K}}\right)^{\perp} \otimes \Omega_{[n+1, \infty)}^{\mathcal{K}}\right) \\
= & \left(\tilde{\mathcal{H}} \otimes \Omega_{\infty}^{\mathcal{K}}\right) \oplus\left(\mathcal{H}^{\circ} \otimes \Omega_{\infty}^{\mathcal{K}}\right) \oplus \mathcal{E} \oplus \bigoplus_{j=1}^{d} V_{j}^{E} \mathcal{E} \oplus \cdots \oplus \bigoplus_{|\alpha|=n-1}^{d} V_{\alpha}^{E} \mathcal{E} .
\end{aligned}
$$

Taking $n \rightarrow \infty$ we have the following:

$$
\mathcal{H} \otimes \mathcal{K}_{\infty}=\left(\tilde{\mathcal{H}} \otimes \Omega_{\infty}^{\mathcal{K}}\right) \oplus\left(\mathcal{H}^{\circ} \otimes \Omega_{\infty}^{\mathcal{K}}\right) \oplus \bigoplus_{\alpha \in \tilde{\Lambda}} V_{\alpha}^{E} \mathcal{E} .
$$

Since $\left(\mathcal{H} \otimes \mathcal{K}_{\infty}\right)^{\circ}=\left(\mathcal{H} \otimes \mathcal{K}_{\infty}\right) \ominus\left(\tilde{\mathcal{H}} \otimes \Omega_{\infty}^{\mathcal{K}}\right)$, it follows that

$$
\left(\mathcal{H} \otimes \mathcal{K}_{\infty}\right)^{\circ}=\mathcal{H}^{\circ} \oplus \bigoplus_{\alpha \in \tilde{\Lambda}} V_{\alpha}^{E} \mathcal{E}
$$

We sum up Propositions 3.3, 3.4 and 3.5 in the following theorem:

Theorem 3.6. For a generalized repeated interaction model involving unitaries $U$ and $\tilde{U}$ as before set $\mathcal{Y}:=\tilde{\mathcal{H}} \otimes\left(\Omega_{1}^{\mathcal{K}}\right)^{\perp} \otimes \Omega_{[2, \infty)}^{\mathcal{K}}$ and $\mathcal{E}:=\mathcal{H} \otimes\left(\Omega_{1}^{\mathcal{K}}\right)^{\perp} \otimes \Omega_{[2, \infty)}^{\mathcal{K}}$. If $\mathcal{E}_{*}:=W^{*} \mathcal{Y}, \mathcal{G}_{*}^{+}:=\bigoplus_{\alpha \in \tilde{\Lambda}} V_{\alpha}^{E} \mathcal{E}_{*}$ and $\mathcal{G}:=\bigoplus_{\alpha \in \tilde{\Lambda}} V_{\alpha}^{E} \mathcal{E}$, then the collection

$$
\left(\left(\mathcal{H} \otimes \mathcal{K}_{\infty}\right)^{\circ}, \underline{V}^{E}=\left(V_{1}^{E}, \ldots, V_{d}^{E}\right), \mathcal{G}_{*}^{+}, \mathcal{G}\right)
$$

is an outgoing Cuntz scattering system such that $\left(\mathcal{H} \otimes \mathcal{K}_{\infty}\right)^{\circ}=\mathcal{H}^{\circ} \oplus \mathcal{G}$.

Remark 3.7. Applying arguments similar to those used for proving the second part of the Proposition 3.5 one can prove the following:

$$
\left(\tilde{\mathcal{H}} \otimes \mathcal{K}_{\infty}\right)^{\circ}=\bigoplus_{\alpha \in \tilde{\Lambda}} V_{\alpha}^{C} \mathcal{Y} .
$$

We refer the reader to Proposition 3.1 of [10] for a result in a similar direction. 


\section{4. $\tilde{\Lambda}$-Linear Systems and Transfer Functions}

We would demonstrate that the outgoing Cuntz scattering system $((\mathcal{H} \otimes$ $\left.\left.\mathcal{K}_{\infty}\right)^{\circ}, \underline{V}^{E}=\left(V_{1}^{E}, \ldots, V_{d}^{E}\right), \mathcal{G}_{*}^{+}, \mathcal{G}\right)$ from Theorem 3.6 has interesting relations with a generalization of the linear systems theory that is associated to our interaction model. For a given model involving unitaries $U$ and $\tilde{U}$ as before, let us define the input space as

$$
\mathcal{U}:=\mathcal{E}=\mathcal{H} \otimes\left(\Omega_{1}^{\mathcal{K}}\right)^{\perp} \otimes \Omega_{[2, \infty)}^{\mathcal{K}} \subset\left(\mathcal{H} \otimes \mathcal{K}_{\infty}\right)^{\circ}
$$

and the output space as

$$
\mathcal{Y}=\tilde{\mathcal{H}} \otimes\left(\Omega_{1}^{\mathcal{K}}\right)^{\perp} \otimes \Omega_{[2, \infty)}^{\mathcal{K}} \subset\left(\tilde{\mathcal{H}} \otimes \mathcal{K}_{\infty}\right)^{\circ} .
$$

Here we assume that a quantum system $\mathcal{A}$ interacts with a stream of copies of another quantum system $\mathcal{B}$ and we assume $\mathcal{H}$ is the (quantum mechanical) Hilbert space of $\mathcal{A}$. Let $\mathcal{K}_{i}$ be the Hilbert space of a part of a stream of copies of $\mathcal{B}$ at time $i$ immediately before the interaction with $\mathcal{A}$. Let the Hilbert space $\mathcal{P}_{i}$ be that the part of a stream of copies of $\mathcal{B}$ at time $i$ immediately after the interaction with $\mathcal{A}$. $\Omega^{\mathcal{K}}$ and $\Omega^{\mathcal{P}}$ denote states indicating that no copy of quantum system $\mathcal{B}$ is present and so no interaction is taking place at time $i$. Then $\eta \in \mathcal{U}=\mathcal{H} \otimes\left(\Omega_{1}^{\mathcal{K}}\right)^{\perp} \otimes \Omega_{[2, \infty)}^{\mathcal{K}} \subset \mathcal{H} \otimes \mathcal{K}_{\infty}$ represents a vector state with copies of quantum system $\mathcal{B}$ arriving at time 1 and stimulating an interaction between the stream of copies of $\mathcal{A}$ and $\mathcal{B}$, but no further copy of $\mathcal{B}$ arriving at later times. But some activity is induced which goes on for a longer period.

Note that $\mathcal{H} \otimes \mathcal{K}=\mathcal{H} \oplus \mathcal{U}$ and $\tilde{\mathcal{H}} \otimes \mathcal{K}=\tilde{\mathcal{H}} \oplus \mathcal{Y}$. So $U$ maps $\mathcal{H} \oplus \mathcal{U}$ onto $\mathcal{H} \otimes \mathcal{P}$ and $\tilde{U}$ maps $\tilde{\mathcal{H}} \oplus \mathcal{Y}$ onto $\tilde{\mathcal{H}} \otimes \mathcal{P}$. Using unitaries $U$ and $\tilde{U}$ we define $F_{j}: \mathcal{H} \rightarrow \mathcal{U}$ and $D_{j}: \tilde{\mathcal{H}} \rightarrow \mathcal{Y}$ for $j=1, \ldots, d$ by

$$
\sum_{j=1}^{d} F_{j}^{*} \eta \otimes \epsilon_{j}:=U(0 \oplus \eta), \sum_{j=1}^{d} D_{j}^{*} y \otimes \epsilon_{j}:=\tilde{U}(0 \oplus y) \text { for } \eta \in \mathcal{U} \text { and } y \in \mathcal{Y} .
$$

Combining equation (4.1) with equations (1.3) and (1.4) we have for $h \in$ $\mathcal{H}, \eta \in \mathcal{U}, \tilde{h} \in \tilde{\mathcal{H}}$ and $y \in \mathcal{Y}$

$$
\begin{gathered}
U(h \oplus \eta)=\sum_{j=1}^{d}\left(E_{j}^{*} h+F_{j}^{*} \eta\right) \otimes \epsilon_{j}, \\
\tilde{U}(\tilde{h} \oplus y)=\sum_{j=1}^{d}\left(C_{j}^{*} \tilde{h}+D_{j}^{*} y\right) \otimes \epsilon_{j}
\end{gathered}
$$

respectively. Using equation (4.3) it can be checked that

$$
\tilde{U}^{*}\left(\tilde{h} \otimes \epsilon_{j}\right)=C_{j} \tilde{h} \oplus D_{j} \tilde{h} \text { for } \tilde{h} \in \tilde{\mathcal{H}} ; j=1, \ldots, d .
$$

Let us define

$$
\tilde{C}:=\sum_{j=1}^{d} D_{j} P_{\tilde{\mathcal{H}}} E_{j}^{*}: \mathcal{H} \rightarrow \mathcal{Y}, \tilde{D}:=\sum_{j=1}^{d} D_{j} P_{\tilde{\mathcal{H}}} F_{j}^{*}: \mathcal{U} \rightarrow \mathcal{Y}
$$


where $P_{\tilde{\mathcal{H}}}$ is the orthogonal projection onto $\tilde{\mathcal{H}}$. It follows that

$$
P_{\mathcal{Y}} \tilde{U}^{*} P_{1} U(h \oplus \eta)=\tilde{C} h+\tilde{D} \eta
$$

where $h \in \mathcal{H}, \eta \in \mathcal{U}, P_{1}$ is as in Proposition 2.1 and $P_{\mathcal{Y}}$ is the orthogonal projection onto $\mathcal{Y}$.

Define a colligation of operators (cf. [3]) using the operators $E_{j}^{*}$ 's, $F_{j}^{*}$ 's, $\tilde{C}$ and $\tilde{D}$ by

$$
\mathcal{C}_{U, \tilde{U}}:=\left(\begin{array}{cc}
E_{1}^{*} & F_{1}^{*} \\
\vdots & \vdots \\
E_{d}^{*} & F_{d}^{*} \\
\tilde{C} & \tilde{D}
\end{array}\right): \mathcal{H} \oplus \mathcal{U} \rightarrow \bigoplus_{j=1}^{d} \mathcal{H} \oplus \mathcal{Y}
$$

From the colligation $\mathcal{C}_{U, \tilde{U}}$ we get the following $\tilde{\Lambda}$-linear system $\sum_{U, \tilde{U}}$ :

$$
\begin{aligned}
x(j \alpha) & =E_{j}^{*} x(\alpha)+F_{j}^{*} u(\alpha), \\
y(\alpha) & =\tilde{C} x(\alpha)+\tilde{D} u(\alpha)
\end{aligned}
$$

where $j=1, \ldots, d$ and $\alpha, j \alpha$ are words in $\tilde{\Lambda}$, and

$$
x: \tilde{\Lambda} \rightarrow \mathcal{H}, u: \tilde{\Lambda} \rightarrow \mathcal{U}, y: \tilde{\Lambda} \rightarrow \mathcal{Y} .
$$

If $x(\emptyset)$ and $u$ are known, then using $\sum_{U, \tilde{U}}$ we can compute $x$ and $y$ recursively. Such a $\tilde{\Lambda}$-linear system is also called a noncommutative Fornasini-Marchesini system in [1] in reference to [6].

Let $z=\left(z_{1}, \ldots, z_{d}\right)$ be a $d$-tuple of formal noncommuting indeterminates. Define the Fourier transforms of $x, u$ and $y$ as

$$
\hat{x}(z)=\sum_{\alpha \in \tilde{\Lambda}} x(\alpha) z^{\alpha}, \hat{u}(z)=\sum_{\alpha \in \tilde{\Lambda}} u(\alpha) z^{\alpha}, \hat{y}(z)=\sum_{\alpha \in \tilde{\Lambda}} y(\alpha) z^{\alpha}
$$

respectively where $z^{\alpha}=z_{\alpha_{n}} \ldots z_{\alpha_{1}}$ for $\alpha=\alpha_{n} \ldots \alpha_{1} \in \tilde{\Lambda}$. Assuming that $z$-variables commute with the coefficients the input-output relation

$$
\hat{y}(z)=\Theta_{U, \tilde{U}}(z) \hat{u}(z)
$$

can be obtained on setting $x(\emptyset):=0$ where

$$
\Theta_{U, \tilde{U}}(z):=\sum_{\alpha \in \tilde{\Lambda}} \Theta_{U, \tilde{U}}^{(\alpha)} z^{\alpha}:=\tilde{D}+\tilde{C} \sum_{\beta \in \tilde{\Lambda}, j=1, \ldots, d}\left(E_{\bar{\beta}}\right)^{*} F_{j}^{*} z^{\beta j} .
$$

Here $\bar{\beta}=\beta_{1} \ldots \beta_{n}$ is the reverse of $\beta=\beta_{n} \ldots \beta_{1} \in \tilde{\Lambda}$ and $\Theta_{U, \tilde{U}}^{(\alpha)}$ maps $\mathcal{U}$ to $\mathcal{Y}$. The formal noncommutative power series $\Theta_{U, \tilde{U}}$ is called the transfer function associated to the unitaries $U$ and $\tilde{U}$. The transfer function is a mathematical tool for encoding the evolution of a $\tilde{\Lambda}$-linear system. For $y(\alpha) \in \mathcal{Y}$ with $\sum_{\alpha \in \tilde{\Lambda}}\|y(\alpha)\|^{2}<\infty$, any series $\sum_{\alpha \in \tilde{\Lambda}} y(\alpha) z^{\alpha}$ stands for a series converging to an element of $\ell^{2}(\tilde{\Lambda}, \mathcal{Y})$. 
Theorem 4.1. The map $M_{\Theta_{U, \tilde{U}}}: \ell^{2}(\tilde{\Lambda}, \mathcal{U}) \rightarrow \ell^{2}(\tilde{\Lambda}, \mathcal{Y})$ defined by

$$
M_{\Theta_{U, \tilde{U}}} \hat{u}(z):=\Theta_{U, \tilde{U}}(z) \hat{u}(z)
$$

is a contraction.

Proof. Observe that $P_{\mathcal{Y}} \tilde{U}^{*} P_{1} U\left(\tilde{h} \otimes \Omega_{\infty}^{\mathcal{K}}\right)=0$ for all $\tilde{h} \in \tilde{\mathcal{H}}$. Consider another colligation which is defined as follows:

$$
\mathcal{C}_{U, \tilde{U}}^{\circ}:=\left(\begin{array}{cc}
E_{1}^{* \circ} & F_{1}^{* \circ} \\
\vdots & \vdots \\
E_{d}^{* \circ} & F_{d}^{* \circ} \\
\tilde{C}^{\circ} & \tilde{D}
\end{array}\right): \mathcal{H}^{\circ} \oplus \mathcal{U} \rightarrow \bigoplus_{j=1}^{d} \mathcal{H}^{\circ} \oplus \mathcal{Y}
$$

where $E_{j}^{* \circ}:=\left.P_{\mathcal{H}^{\circ}} E_{j}^{*}\right|_{\mathcal{H}^{\circ}}: \mathcal{H}^{\circ} \rightarrow \mathcal{H}^{\circ}, F_{j}^{* \circ}:=P_{\mathcal{H}^{\circ}} F_{j}^{*}: \mathcal{U} \rightarrow \mathcal{H}^{\circ}$ and $\tilde{C}^{\circ}:=$ $\left.\tilde{C}\right|_{\mathcal{H}^{\circ}}: \mathcal{H}^{\circ} \rightarrow \mathcal{Y}$ for $j=1, \ldots, d$. Recall that $\mathcal{H}^{\circ}$ and $\left(\mathcal{H} \otimes \mathcal{K}_{\infty}\right)^{\circ}$ were defined in equation array $(2.3)$. Consider the outgoing Cuntz scattering system $((\mathcal{H} \otimes$ $\left.\left.\mathcal{K}_{\infty}\right)^{\circ}, \underline{V}^{E}=\left(V_{1}^{E}, \ldots, V_{d}^{E}\right), \mathcal{G}_{*}^{+}, \mathcal{G}\right)$, with $\left(\mathcal{H} \otimes \mathcal{K}_{\infty}\right)^{\circ}=\mathcal{H}^{\circ} \oplus \mathcal{G}$, constructed by us in Theorem 3.6. In Chapter 5.2 of [3] it is shown that there is an associated unitary colligation

$$
\left(\begin{array}{cc}
\hat{E}_{1} & \hat{F}_{1} \\
\vdots & \vdots \\
\hat{E}_{d} & \hat{F}_{d} \\
\hat{M} & \hat{N}
\end{array}\right): \mathcal{H}^{\circ} \oplus \mathcal{E} \rightarrow \bigoplus_{j=1}^{d} \mathcal{H}^{\circ} \oplus \mathcal{E}_{*}
$$

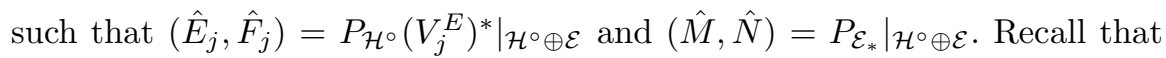
$\mathcal{E}$ and $\mathcal{E}_{*}$ were introduced in Proposition 3.5 and Theorem 3.6 respectively. From equations (4.2) and (4.5) we observe that $\left(E_{j}^{* \circ}, F_{j}^{* \circ}\right)=\left.P_{\mathcal{H}^{\circ} \otimes \epsilon_{j}} U\right|_{\mathcal{H}^{\circ} \oplus \mathcal{E}}$

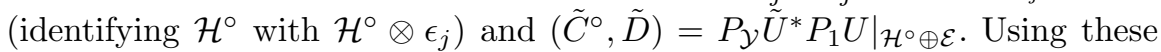
observations we obtain the following relations:

$$
\begin{aligned}
U^{*}\left(E_{j}^{* \circ}, F_{j}^{* \circ}\right) & =\left.U^{*} P_{\mathcal{H}^{\circ} \otimes \epsilon_{j}} U\right|_{\mathcal{H}^{\circ} \oplus \mathcal{E}}=\left.P_{U^{*}\left(\mathcal{H}^{\circ} \otimes \epsilon_{j}\right.}\right|_{\mathcal{H}^{\circ} \oplus \mathcal{E}}=\left.P_{V_{j}^{E} \mathcal{H}^{\circ}}\right|_{\mathcal{H}^{\circ} \oplus \mathcal{E}} \\
& =\left.V_{j}^{E} P_{\mathcal{H}^{\circ}}\left(V_{j}^{E}\right)^{*}\right|_{\mathcal{H}^{\circ} \oplus \mathcal{E}}=V_{j}^{E}\left(\hat{E}_{j}, \hat{F}_{j}\right)
\end{aligned}
$$

for $j=1, \ldots, d$ and

$$
\begin{aligned}
U^{*} \tilde{U}\left(\tilde{C}^{\circ}, \tilde{D}\right) & =\left.U^{*} \tilde{U} P_{\mathcal{Y}} \tilde{U}^{*} P_{1} U\right|_{\mathcal{H}^{\circ} \oplus \mathcal{E}}=\left.U^{*} P_{\tilde{U} \mathcal{Y}} P_{1} U\right|_{\mathcal{H}^{\circ} \oplus \mathcal{E}}=U^{*} P_{\tilde{U} \mathcal{Y}} U_{\mathcal{H}^{\circ} \oplus \mathcal{E}} \\
& =\left.P_{U^{*} \tilde{U} \mathcal{Y}}\right|_{\mathcal{H}^{\circ} \oplus \mathcal{E}}=\left.P_{W^{*} \mathcal{Y}}\right|_{\mathcal{H}^{\circ} \oplus \mathcal{E}} \quad \text { (by equation (3.1)) } \\
& =\left.P_{\mathcal{E}_{*}}\right|_{\mathcal{H}^{\circ} \oplus \mathcal{E}}=(\hat{M}, \hat{N}) .
\end{aligned}
$$

Let $\hat{u}(z)=\sum_{\alpha \in \tilde{\Lambda}} u(\alpha) z^{\alpha} \in \ell^{2}(\tilde{\Lambda}, \mathcal{U})$ with $u(\alpha) \in \mathcal{U}$ such that $\sum_{\alpha \in \tilde{\Lambda}}\|u(\alpha)\|^{2}<\infty$. We would prove that

$$
\left\|M_{\Theta_{U, \tilde{U}}} \hat{u}(z)\right\|^{2} \leq\|\hat{u}(z)\|^{2} .
$$

Define $x: \tilde{\Lambda} \rightarrow \mathcal{H}$ by equation (4.6) such that $x(\emptyset)=0$. Further, define $x^{\circ}(\alpha):=P_{\mathcal{H}^{\circ}} x(\alpha)$ for all $\alpha \in \tilde{\Lambda}$. Now applying the projection $P_{\mathcal{H}^{\circ}}$ to relation 
(4.6) on both sides and using the fact $\tilde{\mathcal{H}}$ is invariant under $E_{j}^{*}$ for $j=1, \ldots, d$ we obtain the following relation:

$$
x^{\circ}(j \alpha)=E_{j}^{* \circ} x^{\circ}(\alpha)+F_{j}^{* \circ} u(\alpha) \text { for all } \alpha \in \tilde{\Lambda}, j=1, \ldots, d .
$$

Because $P_{\mathcal{Y}} \tilde{U}^{*} P_{1} U_{1}\left(\tilde{h} \otimes \Omega_{\infty}^{\mathcal{K}}\right)=0$ for all $\tilde{h} \in \tilde{\mathcal{H}}$ we conclude by equation $(4.5)$ that

$$
\tilde{C} \tilde{h}=0 \text { for } \tilde{h} \in \tilde{\mathcal{H}} .
$$

This implies

$$
\tilde{C} x(\alpha)=\tilde{C}^{\circ} x^{\circ}(\alpha) \text { for all } \alpha \in \tilde{\Lambda} .
$$

Define $y: \tilde{\Lambda} \rightarrow \mathcal{Y}$ by

$$
y(\alpha):=\tilde{C} x(\alpha)+\tilde{D} u(\alpha)
$$

for all $\alpha \in \tilde{\Lambda}$. Recall that the input-output relation stated just before the theorem is

$$
\hat{y}(z)=\sum_{\alpha \in \tilde{\Lambda}} y(\alpha) z^{\alpha}=\Theta_{U, \tilde{U}}(z) \hat{u}(z)\left(=M_{\Theta_{U, \tilde{U}}} \hat{u}(z)\right) .
$$

Using the unitary colligation given in equation (4.9) we have

$$
\begin{aligned}
\left\|x^{\circ}(\alpha)\right\|^{2}+\|u(\alpha)\|^{2} & =\sum_{j=1}^{d}\left\|\hat{E}_{j} x^{\circ}(\alpha)+\hat{F}_{j} u(\alpha)\right\|^{2}+\left\|\hat{M} x^{\circ}(\alpha)+\hat{N} u(\alpha)\right\|^{2} \\
& =\sum_{j=1}^{d}\left\|E_{j}^{* \circ} x^{\circ}(\alpha)+F_{j}^{* \circ} u(\alpha)\right\|^{2}+\left\|\tilde{C}^{\circ} x^{\circ}(\alpha)+\tilde{D} u(\alpha)\right\|^{2} \\
& =\sum_{j=1}^{d}\left\|x^{\circ}(j \alpha)\right\|^{2}+\|\tilde{C} x(\alpha)+\tilde{D} u(\alpha)\|^{2} \\
& =\sum_{j=1}^{d}\left\|x^{\circ}(j \alpha)\right\|^{2}+\|y(\alpha)\|^{2}
\end{aligned}
$$

for all $\alpha \in \tilde{\Lambda}$. In the above calculation equations (4.10), (4.11), (4.12), (4.14) and (4.15) respectively have been used. This gives us

$$
\|u(\alpha)\|^{2}-\|y(\alpha)\|^{2}=\sum_{j=1}^{d}\left\|x^{\circ}(j \alpha)\right\|^{2}-\left\|x^{\circ}(\alpha)\right\|^{2}
$$

for all $\alpha \in \tilde{\Lambda}$. Summing over all $\alpha \in \tilde{\Lambda}$ with $|\alpha| \leq n$ and using the fact that $x^{\circ}(\emptyset)=0$ we obtain

$$
\sum_{|\alpha| \leq n}\|u(\alpha)\|^{2}-\sum_{|\alpha| \leq n}\|y(\alpha)\|^{2}=\sum_{|\alpha|=n+1}\left\|x^{\circ}(\alpha)\right\|^{2} \geq 0 \text { for all } n \in \mathbb{N} .
$$

Therefore

$$
\sum_{|\alpha| \leq n}\|y(\alpha)\|^{2} \leq \sum_{|\alpha| \leq n}\|u(\alpha)\|^{2} \text { for all } n \in \mathbb{N}
$$

Finally taking limit $n \rightarrow \infty$ both the sides we get that $M_{\Theta_{U, \tilde{U}}}$ is a contraction. 
$M_{\Theta_{U, \tilde{U}}}$ is a multi-analytic operator ([15]) (also called analytic intertwining operator in [3]) because

$$
M_{\Theta_{U, \tilde{U}}}\left(\sum_{\alpha \in \tilde{\Lambda}} u(\alpha) z^{\alpha} z^{j}\right)=M_{\Theta_{U, \tilde{U}}}\left(\sum_{\alpha \in \tilde{\Lambda}} u(\alpha) z^{\alpha}\right) z^{j} \text { for } j=1, \ldots, d,
$$

i.e., $M_{\Theta_{U, \tilde{U}}}$ intertwines with right translation. The noncommutative power series $\Theta_{U, \tilde{U}}$ is called the symbol of $M_{\Theta_{U, \tilde{U}}}$.

\section{Transfer Functions, Observability and Scattering}

We would now establish that the transfer function can be derived from the coisometry $W$ of section 2 . In the last section $d$-tuple $z=\left(z_{1}, \ldots, z_{d}\right)$ of formal noncommuting indeterminates were employed. Treat $\left(z^{\alpha}\right)_{\alpha \in \tilde{\Lambda}}$ as an orthonormal basis of $\ell^{2}(\tilde{\Lambda}, \mathbb{C})$. Assume $\mathcal{Y}$ and $\mathcal{U}$ to be the spaces associated with our model with unitaries $U$ and $\tilde{U}$ as in the last section. It follows from Remark 3.7 that there exist a unitary operator $\tilde{\Gamma}:\left(\tilde{\mathcal{H}} \otimes \mathcal{K}_{\infty}\right)^{\circ} \rightarrow \ell^{2}(\tilde{\Lambda}, \mathcal{Y})$ defined by

$$
\tilde{\Gamma}\left(V_{\alpha}^{C} y\right):=y z^{\bar{\alpha}} \text { for all } \alpha \in \tilde{\Lambda}, y \in \mathcal{Y} .
$$

We observe the following intertwining relation:

$$
\tilde{\Gamma}\left(V_{\alpha}^{C} y\right)=(\tilde{\Gamma} y) z^{\bar{\alpha}}
$$

Similarly, using Theorem 3.6, we can define a unitary operator $\Gamma:(\mathcal{H} \otimes$ $\left.\mathcal{K}_{\infty}\right)^{\circ}\left(=\left(\mathcal{H}^{\circ} \oplus \mathcal{G}\right)\right) \rightarrow \mathcal{H}^{\circ} \oplus \ell^{2}(\tilde{\Lambda}, \mathcal{U})$ by

$$
\Gamma\left(\stackrel{\circ}{h} \oplus V_{\alpha}^{E} \eta\right):=\stackrel{\circ}{h} \oplus \eta z^{\bar{\alpha}} \text { for all } \alpha \in \tilde{\Lambda}
$$

where $\stackrel{\circ}{h} \mathcal{H}^{\circ}, \eta \in \mathcal{U}$. In this case the intertwining relation is

$$
\Gamma\left(V_{\alpha}^{E} \eta\right)=(\Gamma \eta) z^{\bar{\alpha}} .
$$

Using the coisometric operator $W$, which appears in Remark 2.3, we define $\Gamma_{W}$ by the following commutative diagram:

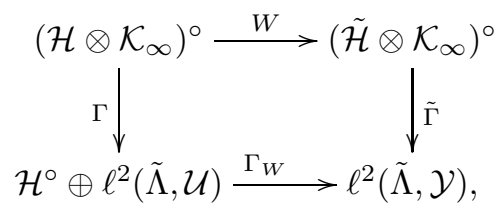

i.e., $\Gamma_{W}=\tilde{\Gamma} W \Gamma^{-1}$.

Theorem 5.1. $\Gamma_{W}$ defined by the above commutative diagram satisfies

$$
\left.\Gamma_{W}\right|_{\ell^{2}(\tilde{\Lambda}, \mathcal{U})}=M_{\Theta_{U, \tilde{U}}} .
$$

Proof. Using the intertwining relation $V_{j}^{C} W=W V_{j}^{E}$ from Remark 2.3, and equations (5.1) and (5.2) we obtain

$$
\begin{aligned}
\Gamma_{W}\left(\eta z^{\beta} z^{j}\right) & =\tilde{\Gamma} W \Gamma^{-1}\left(\eta z^{\beta} z^{j}\right)=\tilde{\Gamma} W V_{j}^{E} V_{\bar{\beta}}^{E} \eta \\
& =\tilde{\Gamma} V_{j}^{C} V_{\bar{\beta}}^{C} W \eta=(\tilde{\Gamma} W \eta) z^{\beta} z^{j}=\Gamma_{W}\left(\eta z^{\beta}\right) z^{j}
\end{aligned}
$$


for $\eta \in \mathcal{U}, \beta \in \tilde{\Lambda}, j=1, \ldots, d$. Hence, $\left.\Gamma_{W}\right|_{\ell^{2}(\tilde{\Lambda}, \mathcal{U})}$ is a multi-analytic operator. For computing its symbol we determine $\Gamma_{W} \eta$ for $\eta \in \mathcal{U}$, where $\eta$ is identified with $\eta z^{\phi} \in \ell^{2}(\tilde{\Lambda}, \mathcal{U})$. For $\alpha=\alpha_{n-1} \ldots \alpha_{1} \in \tilde{\Lambda}$ let $P_{\alpha}$ be the orthogonal projection onto

$$
\begin{aligned}
& \tilde{\Gamma}^{-1}\left\{f \in \ell^{2}(\tilde{\Lambda}, \mathcal{Y}): f=y z^{\alpha} \text { for some } y \in \mathcal{Y}\right\} \\
= & V_{\bar{\alpha}}^{C} \mathcal{Y}=\tilde{U}_{1}^{*} \ldots \tilde{U}_{n-1}^{*}\left(\tilde{\mathcal{H}} \otimes \epsilon_{\alpha_{1}} \otimes \cdots \otimes \epsilon_{\alpha_{n-1}} \otimes\left(\Omega_{n}^{\mathcal{K}}\right)^{\perp} \otimes \Omega_{[n+1, \infty)}^{\mathcal{K}}\right)
\end{aligned}
$$

with $\tilde{U}_{i}$ 's as in Proposition 2.1.

Recall that the tuple $\underline{E}$ associated with the unitary $U$ is a lifting of the tuple $\underline{C}$ (associated with the unitary $\tilde{U}$ ) and so $\underline{E}$ can be written as a block matrix in terms of $\underline{C}$ as follows: $E_{j}=\left(\begin{array}{cc}C_{j} & 0 \\ B_{j} & A_{j}\end{array}\right)$ for $j=1, \ldots, d$ w.r.t. to the decomposition $\mathcal{H}=\tilde{\mathcal{H}} \oplus \mathcal{H}^{\circ}$ where $\underline{B}$ and $\underline{A}$ are some row contractions. Because $\underline{E}$ is a coisometric lifting of $\underline{C}$ we have

$$
\sum_{j=1}^{d} C_{j} C_{j}^{*}=I \text { and } \sum_{j=1}^{d} C_{j} B_{j}^{*}=0
$$

(cf. [5]) . Now using these relations and equations (4.2), (4.3) and (4.4) it can be easily verified that $P_{\alpha} \tilde{U}_{1}^{*} \ldots \tilde{U}_{n}^{*} P_{n} U_{n} \ldots U_{1} \eta=P_{\alpha} \tilde{U}_{1}^{*} \ldots \tilde{U}_{m}^{*} P_{m} U_{m} \ldots U_{1} \eta$ for all $m \geq n, \eta \in \mathcal{U}$. Using the formula of $W$ from Proposition 2.1 we obtain

$$
P_{\alpha} W \eta=P_{\alpha} \tilde{U}_{1}^{*} \ldots \tilde{U}_{n}^{*} P_{n} U_{n} \ldots U_{1} \eta \text { for } \eta \in \mathcal{U} .
$$

Finally for $\eta \in \mathcal{U}$

$$
P_{\alpha} \tilde{U}_{1}^{*} \ldots \tilde{U}_{n}^{*} P_{n} U_{n} \ldots U_{1} \eta=\left\{\begin{array}{l}
\tilde{D} \eta \text { if } n=1, \alpha=\emptyset \\
V_{\bar{\alpha}}^{C}\left(\tilde{C} E_{\alpha_{n-1}}^{*} \ldots E_{\alpha_{2}}^{*} F_{\alpha_{1}}^{*} \eta\right) \text { if } n=|\alpha|+1 \geq 2 .
\end{array}\right.
$$

This implies for $\eta \in \mathcal{U}$

$$
\tilde{\Gamma} W \Gamma^{-1} \eta=\tilde{\Gamma} W \eta=\tilde{D} \eta \oplus \sum_{|\alpha| \geq 1}\left(\tilde{C} E_{\alpha_{n-1}}^{*} \ldots E_{\alpha_{2}}^{*} F_{\alpha_{1}}^{*} \eta\right) z^{\alpha} .
$$

Comparing this with equation (4.8) we conclude that $\left.\Gamma_{W}\right|_{\ell^{2}(\tilde{\Lambda}, \mathcal{U})}=M_{\Theta_{U, \tilde{U}}}$.

Note that the Theorem 4.1 and its proof concern the transfer function of the $\tilde{\Lambda}$-linear system and has nothing to do with the scattering theory. Theorem 5.1, on the other hand, is the scattering theory part in the sense of Lax-Phillips [12. The same function $M_{\Theta_{U, \tilde{U}}}$ relates the outgoing Fourier representation for a vector in the ambient scattering Hilbert space to the incoming Fourier representation for the same vector. This makes $M_{\Theta_{U, \tilde{U}}}$ the scattering function for the outgoing Cuntz scattering system. We introduce a notion from the linear systems theory for our model:

Definition 5.2. The observability operator $W_{0}: \mathcal{H}^{\circ} \rightarrow \ell^{2}(\tilde{\Lambda}, \mathcal{Y})$ is defined as the restriction of the operator $\Gamma_{W}$ to $\mathcal{H}^{\circ}$, i.e., $W_{0}=\left.\Gamma_{W}\right|_{\mathcal{H}^{\circ}}$. 
It follows that $W_{0} \stackrel{\circ}{h}=\left(\tilde{C}\left(E_{\bar{\alpha}}\right)^{*} h\right)_{\alpha \in \tilde{\Lambda}}$. Popescu has studied the similar types of operators called Poisson kernels in [16.

Definition 5.3. If there exist $k, K>0$ such that for all $\stackrel{\circ}{h} \in \mathcal{H}^{\circ}$

$$
k\|\stackrel{\circ}{h}\|^{2} \leq \sum_{\alpha \in \tilde{\Lambda}}\left\|\tilde{C}\left(E_{\bar{\alpha}}\right)^{*} \stackrel{\circ}{h}\right\|^{2}=\left\|W_{0} \stackrel{\circ}{h}\right\|^{2} \leq K\|\stackrel{\circ}{h}\|^{2},
$$

then the $\tilde{\Lambda}$-linear system is called (uniformly) observable.

We illustrate below that the notion of observability is closely related to the scattering theory notions of noncommutative Markov chains. Observability of a system for $\operatorname{dim} \mathcal{H}<\infty$ is interpreted as the property of the system that in the absence of $\mathcal{U}$-inputs we can determine the original state $h \in \mathcal{H}^{\circ}$ of the system from all $\mathcal{Y}$-outputs at all times. Uniform observability is an analog of this for $\operatorname{dim} \mathcal{H}=\infty$.

We extend $W_{0}$ to

$$
\widehat{W}_{0}:\left(\tilde{\mathcal{H}} \oplus \mathcal{H}^{\circ}\right)(=\mathcal{H}) \longrightarrow \tilde{\mathcal{H}} \oplus \ell^{2}(\tilde{\Lambda}, \mathcal{Y})
$$

by defining $\widehat{W}_{0} \tilde{h}:=\tilde{h}$ for all $\tilde{h} \in \tilde{\mathcal{H}}$. If $W_{0}$ is uniformly observable, then using $\hat{k}=k$ and $\hat{K}=\max \{1, K\}$ the above inequalities can be extended to $\widehat{W}_{0}$ on $\mathcal{H}$ as

$$
\hat{k}\|h\|^{2} \leq\left\|\widehat{W}_{0} h\right\|^{2} \leq \hat{K}\|h\|^{2}
$$

for all $h \in \mathcal{H}$.

Before stating the main theorem of this section regarding observability we recall from [5] the following: Let $\underline{C}$ be a row contraction on a Hilbert space $\mathcal{H}_{C}$. The lifting $\underline{E}$ of $\underline{C}$ is called subisometric 5] if the minimal isometric dilations $\widehat{V}^{E}$ and $\underline{V}^{C}$ of $\underline{E}$ and $\underline{C}$ respectively are unitarily equivalent and the corresponding unitary, which intertwines between $\widehat{V}_{i}^{E}$ and $\widehat{V}_{i}^{C}$ for all $i=1,2, \ldots, d$, acts as identity on $\mathcal{H}_{C}$. Some of the techniques used here are from the scattering theory of noncommutative Markov chains (cf. [11], [8]).

Theorem 5.4. For any $\tilde{\Lambda}$-linear system associated to a generalized repeated interaction model with unitaries $U, \tilde{U}$ the following statements are equivalent:

(a) The system is (uniformly) observable.

(b) The observability operator $W_{0}$ is isometric.

(c) The tuple $\underline{E}$ associated with the unitary $U$ is a subisometric lifting of the tuple $\underline{C}$ (associated with the unitary $\tilde{U})$.

(d) $W:\left(\mathcal{H} \otimes \mathcal{K}_{\infty}\right)^{\circ} \rightarrow\left(\tilde{\mathcal{H}} \otimes \mathcal{K}_{\infty}\right)^{\circ}$ is unitary.

If one of the above holds, then

(e) The transfer function $\Theta_{U, \tilde{U}}$ is inner, i.e., $M_{\Theta_{U, \tilde{U}}}: \ell^{2}(\tilde{\Lambda}, \mathcal{U}) \rightarrow \ell^{2}(\tilde{\Lambda}, \mathcal{Y})$ is isometric.

If we have additional assumptions, viz. $\operatorname{dim} \mathcal{H}<\infty$ and $\operatorname{dim} \mathcal{P} \geq 2$, then the converse holds, i.e., (e) implies all of $(a),(b),(c)$ and $(d)$. 
Proof. Clearly $(d) \Rightarrow(b) \Rightarrow(a)$. We now prove $(a) \Rightarrow(d)$. Because the system is (uniformly) observable there exist $k>0$ such that for all $\stackrel{\circ}{h} \in \mathcal{H}^{\circ}$

$$
k\|\stackrel{\circ}{h}\|^{2} \leq\left\|W_{0} \stackrel{\circ}{h}\right\|^{2} .
$$

Since $\bigcup_{m \geq 1} \mathcal{H} \otimes \mathcal{K}_{[1, m]}$ is a dense subspace of $\mathcal{H} \otimes \mathcal{K}_{\infty}$, for any $0 \neq \eta \in \mathcal{H} \otimes \mathcal{K}_{\infty}$ there exist $n \in \mathbb{N}$ and $\eta^{\prime} \in \mathcal{H} \otimes \mathcal{K}_{[1, n]}$ such that

$$
\left\|\eta-\eta^{\prime}\right\|<\frac{\sqrt{k}}{\sqrt{k}+1}\|\eta\| .
$$

Let $\eta_{0} \in \mathcal{H} \otimes \mathcal{K}_{[1, n]}$. Suppose $U_{n} \ldots U_{1} \eta_{0}=h_{0} \otimes p_{0} \otimes \Omega_{[n+1, \infty)}^{\mathcal{K}}$, where $h_{0} \in \mathcal{H}$, $p_{0} \in \mathcal{P}_{[1, n]}$. Then clearly

$$
\lim _{N \rightarrow \infty}\left\|\tilde{U}_{1}^{*} \ldots \tilde{U}_{n}^{*} \tilde{U}_{n+1}^{*} \ldots \tilde{U}_{N}^{*} P_{N} U_{N} \ldots U_{n+1} U_{n} \ldots U_{1} \eta_{0}\right\|=\left\|\widehat{W}_{0} h_{0}\right\|\left\|p_{0}\right\|
$$

and thus by Proposition 2.1 it is equal to $\left\|\widehat{W} \eta_{0}\right\|$. Because the system is (uniformly) observable,

$$
\left\|\widehat{W}_{0} h_{0}\right\|\left\|p_{0}\right\| \geq \sqrt{k}\left\|h_{0}\right\|\left\|p_{0}\right\| .
$$

Therefore $\left\|\widehat{W} \eta_{0}\right\|^{2} \geq k\left\|\eta_{0}\right\|^{2}$. However, in general $U_{n} \ldots U_{1} \eta_{0}=\sum_{j} h_{0}^{(j)} \otimes$ $p_{0}^{(j)} \otimes \Omega_{[n+1, \infty)}^{\mathcal{K}}$ with $h_{0}^{(j)} \in \mathcal{H}$ and some mutually orthogonal vectors $p_{0}^{(j)} \in$ $\mathcal{P}_{[1, n]}$. By using the above inequality for each term of the summation and then adding them we find that in general for all $\eta_{0} \in \mathcal{H} \otimes \mathcal{K}_{[1, n]}$

$$
\left\|\widehat{W} \eta_{0}\right\|^{2} \geq k\left\|\eta_{0}\right\|^{2}
$$

In particular, for $\eta^{\prime} \in \mathcal{H} \otimes \mathcal{K}_{[1, n]}$ we have the above inequality. Therefore

$$
\begin{aligned}
\|\widehat{W} \eta\| & \geq\left\|\widehat{W} \eta^{\prime}\right\|-\left\|\widehat{W}\left(\eta^{\prime}-\eta\right)\right\| \\
& \geq \sqrt{k}\left\|\eta^{\prime}\right\|-\left\|\eta-\eta^{\prime}\right\| \\
& \geq \sqrt{k}\|\eta\|-(\sqrt{k}+1)\left\|\eta-\eta^{\prime}\right\|>0 .
\end{aligned}
$$

This implies $\widehat{W} \eta \neq 0$ for all $0 \neq \eta \in \mathcal{H} \otimes \mathcal{K}_{\infty}$ and hence $\widehat{W}$ is injective. Recall that $\widehat{W}$ is a coisometry and an injective coisometry is unitary. Further, because $\widehat{W}\left(\tilde{h} \otimes \Omega_{\infty}^{\mathcal{K}}\right)=\tilde{h} \otimes \Omega_{\infty}^{\mathcal{K}}$ for all $\tilde{h} \in \tilde{\mathcal{H}}$ it follows that $W$ is unitary. This establishes $(a) \Rightarrow(d)$ and we have proved $(a) \Leftrightarrow(b) \Leftrightarrow(d)$.

Next we prove $(d) \Leftrightarrow(c)$. Assume that $(d)$ holds. Since $W$ is unitary, clearly $\widehat{W}$ is unitary. We know that $\widehat{W}$ intertwines between the minimal isometric dilations $\underline{\widehat{V}}^{E}$ and $\widehat{\widehat{V}}^{C}$ of $\underline{E}$ and $\underline{C}$ respectively. Hence $\underline{E}$ is a subisometric lifting of $\underline{C}$.

Conversely, if we assume $(c)$, then by the definition of subisometric lifting there exist a unitary operator

$$
\widehat{W}_{1}: \mathcal{H} \otimes \mathcal{K}_{\infty} \longrightarrow \tilde{\mathcal{H}} \otimes \mathcal{K}_{\infty}
$$

which intertwines between $\underline{V}^{E}$ and $\widehat{V}^{C}$, and $\widehat{W}_{1}$ acts as an identity on $\tilde{\mathcal{H}} \otimes \Omega_{\infty}^{\mathcal{K}}$. To prove $W$ is unitary it is enough to prove $\widehat{W}$ is unitary. We show that $\widehat{W}=\widehat{W}_{1}$. By the definition of the minimal isometric dilation we know that 
$\tilde{\mathcal{H}} \otimes \mathcal{K}_{\infty}=\overline{\operatorname{span}}\left\{\widehat{V}_{\alpha}^{C}\left(\tilde{h} \otimes \Omega_{\infty}^{\mathcal{K}}\right): \tilde{h} \in \tilde{\mathcal{H}}, \alpha \in \tilde{\Lambda}\right\}$. For $j=1, \ldots, d$ and $\tilde{h} \in \tilde{\mathcal{H}}$, by equation (2.2) and Proposition 2.2,

$$
\begin{aligned}
\widehat{W}^{*} \widehat{V}_{j}^{C}\left(\tilde{h} \otimes \Omega_{\infty}^{\mathcal{K}}\right) & =\widehat{V}_{j}^{E} \widehat{W}^{*}\left(\tilde{h} \otimes \Omega_{\infty}^{\mathcal{K}}\right)=\widehat{V}_{j}^{E}\left(\tilde{h} \otimes \Omega_{\infty}^{\mathcal{K}}\right) \\
& =\widehat{W}_{1}^{*} \widehat{V}_{j}^{C} \widehat{W}_{1}\left(\tilde{h} \otimes \Omega_{\infty}^{\mathcal{K}}\right)=\widehat{W}_{1}^{*} \widehat{V}_{j}^{C}\left(\tilde{h} \otimes \Omega_{\infty}^{\mathcal{K}}\right) .
\end{aligned}
$$

Thus $\widehat{W}^{*}=\widehat{W}_{1}^{*}$ and hence $\widehat{W}=\widehat{W}_{1}$.

To prove $(d) \Rightarrow(e)$ we at first note that since $W$ is unitary, $\Gamma_{W}$ is also unitary. By Theorem 4.2, we have $M_{\Theta_{U, \tilde{U}}}=\left.\Gamma_{W}\right|_{\ell^{2}(\tilde{\Lambda}, \mathcal{U})}$. Since a restriction of a unitary operator is an isometry, $M_{\Theta_{U, \tilde{U}}}$ is isometric.

Finally with the additional assumptions $\operatorname{dim} \mathcal{H}<\infty$ and $\operatorname{dim} \mathcal{P} \geq 2$, we show $(e) \Rightarrow(b)$. Define

$$
\mathcal{H}_{\text {scat }}:=\mathcal{H} \cap \widehat{W}^{*}\left(\tilde{\mathcal{H}} \otimes \mathcal{K}_{\infty}\right)=\tilde{\mathcal{H}} \oplus\left\{\stackrel{\circ}{h} \in \mathcal{H}^{\circ}:\left\|W_{0} \stackrel{\circ}{h}\right\|=\|\stackrel{\circ}{h}\|\right\} .
$$

Since $\left\|\widehat{W}_{0} h\right\|=\lim _{n \rightarrow \infty}\left\|\tilde{U}_{1} \ldots \tilde{U}_{n} \tilde{P}_{n} U_{n} \ldots U_{1} h\right\|$ by Proposition 2.1 , the following can be easily verified:

$$
U\left(\mathcal{H}_{\text {scat }} \otimes \Omega^{\mathcal{K}}\right) \subset \mathcal{H}_{\text {scat }} \otimes \mathcal{P} .
$$

Because $M_{\Theta_{U, \tilde{U}}}=\left.\Gamma_{W}\right|_{\ell^{2}(\tilde{\Lambda}, \mathcal{U})}$ is isometric by (e), it can be checked that

$$
U\left(\mathcal{H} \otimes\left(\Omega^{\mathcal{K}}\right)^{\perp}\right) \subset \mathcal{H}_{\text {scat }} \otimes \mathcal{P} .
$$

Combining equations (5.4) and (5.5) we have

$$
U^{*}\left(\left(\mathcal{H} \ominus \mathcal{H}_{\text {scat }}\right) \otimes \mathcal{P}\right) \subset\left(\mathcal{H} \ominus \mathcal{H}_{\text {scat }}\right) \otimes \Omega^{\mathcal{K}} .
$$

Since $\operatorname{dim} \mathcal{H}<\infty$ and $\operatorname{dim} \mathcal{P} \geq 2$, we obtain $\mathcal{H} \ominus \mathcal{H}_{\text {scat }}=\{0\}$, i.e., $\mathcal{H}=\mathcal{H}_{\text {scat }}$. This implies $W_{0}$ is isometric and hence $(e) \Rightarrow(b)$.

\section{Transfer Functions and Characteristic Functions of Liftings}

Continuing with the study of our generalized repeated interaction model, from equations (2.1) and (4.4) we obtain

$$
\widehat{V}_{j}^{C}\left(\tilde{h} \otimes \Omega_{\infty}^{\mathcal{K}}\right)=\left(C_{j} \tilde{h} \oplus D_{j} \tilde{h}\right) \otimes \Omega_{[2, \infty)}^{\mathcal{K}} \text { for } \tilde{h} \in \tilde{\mathcal{H}} \text { and } j=1, \ldots, d .
$$

Let $D_{C}:=\left(I-\underline{C}^{*} \underline{C}\right)^{\frac{1}{2}}: \bigoplus_{i=1}^{d} \tilde{\mathcal{H}} \rightarrow \bigoplus_{i=1}^{d} \tilde{\mathcal{H}}$ denote the defect operator and $\mathcal{D}_{C}:=\overline{\text { Range } D_{C}}$. The full Fock space over $\mathbb{C}^{d}(d \geq 2)$ denoted by $\mathcal{F}$ is

$$
\mathcal{F}=\mathbb{C} \oplus \mathbb{C}^{d} \oplus\left(\mathbb{C}^{d}\right)^{\otimes^{2}} \oplus \cdots \oplus\left(\mathbb{C}^{d}\right)^{\otimes^{m}} \oplus \cdots .
$$

The vector $e_{\emptyset}:=1 \oplus 0 \oplus \cdots$ is called the vacuum vector. Let $\left\{e_{1}, \ldots, e_{d}\right\}$ be the standard orthonormal basis of $\mathbb{C}^{d}$. For $\alpha \in \tilde{\Lambda}$ and $|\alpha|=n, e_{\alpha}$ denote the vector $e_{\alpha_{1}} \otimes e_{\alpha_{2}} \otimes \cdots \otimes e_{\alpha_{n}}$ in the full Fock space $\mathcal{F}$. We recall that Popescu's construction [13] of the minimal isometric dilation $\tilde{V}^{C}=\left(\tilde{V}_{1}^{C}, \ldots, \tilde{V}_{d}^{C}\right)$ on $\tilde{\mathcal{H}} \oplus\left(\mathcal{F} \otimes \mathcal{D}_{C}\right)$ of the tuple $\underline{C}$ is

$$
\tilde{V}_{j}^{C}\left(\tilde{h} \oplus \sum_{\alpha \in \tilde{\Lambda}} e_{\alpha} \otimes d_{\alpha}\right)=C_{j} \tilde{h} \oplus\left[e_{\emptyset} \otimes\left(D_{C}\right)_{j} \tilde{h}+e_{j} \otimes \sum_{\alpha \in \tilde{\Lambda}} e_{\alpha} \otimes d_{\alpha}\right]
$$


for $\tilde{h} \in \tilde{\mathcal{H}}$ and $d_{\alpha} \in \mathcal{D}_{C}$ where $\left(D_{C}\right)_{j} \tilde{h}=D_{C}(0, \ldots, \tilde{h}, \ldots, 0)(\tilde{h}$ is embedded at the $j^{\text {th }}$ component). So

$$
\tilde{V}_{j}^{C} \tilde{h}=C_{j} \tilde{h} \oplus\left(e_{\emptyset} \otimes\left(D_{C}\right)_{j} \tilde{h}\right) \text { for } \tilde{h} \in \tilde{\mathcal{H}} \text { and } j=1, \ldots, d .
$$

From equations (6.1) and (6.2) it follows that

$$
\left\|\sum_{j=1}^{d} D_{j} \tilde{h}_{j}\right\|^{2}=\left\|\sum_{j=1}^{d}\left(D_{C}\right)_{j} \tilde{h}_{j}\right\|^{2}
$$

where $\tilde{h}_{j} \in \tilde{\mathcal{H}}$ for $j=1, \ldots, d$. Let $\Phi_{C}: \overline{\operatorname{span}}\left\{D_{j} \tilde{h}: \tilde{h} \in \tilde{\mathcal{H}}, j=1, \ldots, d\right\} \rightarrow$ $\mathcal{D}_{C}$ be the unitary given by

$$
\Phi_{C}\left(\sum_{j=1}^{d} D_{j} \tilde{h}_{j}\right)=\sum_{j=1}^{d}\left(D_{C}\right)_{j} \tilde{h}_{j} \text { for } \tilde{h}_{j} \in \tilde{\mathcal{H}} \text { and } j=1, \ldots, d .
$$

Similarly for $E_{i}$ 's and $F_{i}$ 's obtained from interaction $U$ in equation (4.2) we set $D_{E}:=\left(I-\underline{E}^{*} \underline{E}\right)^{\frac{1}{2}}: \bigoplus_{i=1}^{d} \mathcal{H} \rightarrow \bigoplus_{i=1}^{d} \mathcal{H}$ and $\mathcal{D}_{E}:=\overline{\text { Range } D_{E}}$, and define another unitary operator $\Phi_{E}: \overline{\operatorname{span}}\left\{F_{j} h: h \in \mathcal{H}, j=1, \ldots, d\right\} \rightarrow \mathcal{D}_{E}$ by

$$
\Phi_{E}\left(\sum_{j=1}^{d} F_{j} h_{j}\right)=\sum_{j=1}^{d}\left(D_{E}\right)_{j} h_{j} \text { for } h_{j} \in \mathcal{H} \text { and } j=1, \ldots, d .
$$

The second equation of (4.1) yields

$$
\sum_{j=1}^{d} D_{j} D_{j}^{*} y=y \text { for } y \in \mathcal{Y} .
$$

This implies

$$
\overline{\operatorname{span}}\left\{D_{j} \tilde{h}: \tilde{h} \in \tilde{\mathcal{H}}, j=1, \ldots, d\right\}=\mathcal{Y} .
$$

Similarly, we can show that $\overline{\operatorname{span}}\left\{F_{j} h: h \in \mathcal{H}, j=1, \ldots, d\right\}=\mathcal{U}$. Thus $\Phi_{C}$ is a unitary from $\mathcal{Y}$ onto $\mathcal{D}_{C}$ and $\Phi_{E}$ is a unitary from $\mathcal{U}$ onto $\mathcal{D}_{E}$. As a consequence we have for $i, j=1, \ldots, d$

$$
\begin{aligned}
D_{j}^{*} D_{i} & =\left(D_{C}\right)_{j}^{*}\left(D_{C}\right)_{i}=\delta_{i j} I-C_{j}^{*} C_{i}, \\
F_{j}^{*} F_{i} & =\left(D_{E}\right)_{j}^{*}\left(D_{E}\right)_{i}=\delta_{i j} I-E_{j}^{*} E_{i} .
\end{aligned}
$$

Define unitaries $\tilde{M}_{\Phi_{C}}: \ell^{2}(\tilde{\Lambda}, \mathcal{Y}) \rightarrow \mathcal{F} \otimes \mathcal{D}_{C}$ and $\tilde{\Phi}_{E}: \mathcal{U} z^{\emptyset} \rightarrow e_{\emptyset} \otimes \mathcal{D}_{E}$ by

$$
\begin{aligned}
\tilde{M}_{\Phi_{C}}\left(\sum_{\alpha \in \tilde{\Lambda}} y_{\alpha} z^{\alpha}\right) & :=\sum_{\alpha \in \tilde{\Lambda}} e_{\bar{\alpha}} \otimes \Phi_{C}\left(y_{\alpha}\right), \\
\tilde{\Phi}_{E}\left(u z^{\emptyset}\right) & :=e_{\emptyset} \otimes \Phi_{E} u
\end{aligned}
$$

which would be useful in comparing transfer functions with characteristic functions.

Define $D_{*, A}:=\left(I-\underline{A A}^{*}\right)^{\frac{1}{2}}: \mathcal{H}^{\circ} \rightarrow \mathcal{H}^{\circ}$ and $\mathcal{D}_{*, A}:=\overline{\text { Range } D_{*, A}}$. Because $\underline{E}$ is a coisometric lifting of $\underline{C}$, using Theorem 2.1 of $[\underline{5}$ ] we conclude 
that there exist an isometry $\gamma: \mathcal{D}_{*, A} \rightarrow \mathcal{D}_{C}$ with $\gamma D_{*, A} h=\underline{B}^{*} h$ for all $h \in \mathcal{H}^{\circ}$. Further, for $h \in \mathcal{H}^{\circ}$

$$
\begin{aligned}
\Phi_{C} \tilde{C} h & =\Phi_{C} \sum_{j=1}^{d} D_{j} P_{\tilde{\mathcal{H}}} E_{j}^{*} h=\Phi_{C} \sum_{j=1}^{d} D_{j} P_{\tilde{\mathcal{H}}}\left(B_{j}^{*} h \oplus A_{j}^{*} h\right) \\
& =\Phi_{C} \sum_{j=1}^{d} D_{j} B_{j}^{*} h=\sum_{j=1}^{d}\left(D_{C}\right)_{j} B_{j}^{*} h \\
& =D_{C} \underline{B}^{*} h=\underline{B}^{*} h .
\end{aligned}
$$

The last equality holds because for the coisometric tuple $\underline{C}$ the operator $D_{C}$ is the projection onto $\mathcal{D}_{C}$ and Range $\underline{B}^{*} \subset \mathcal{D}_{C}$. This implies

$$
\Phi_{C} \tilde{C} h=\gamma D_{*, A} h
$$

The characteristic function $M_{C, E}: \mathcal{F} \otimes \mathcal{D}_{E} \rightarrow \mathcal{F} \otimes \mathcal{D}_{C}$ of lifting $\underline{E}$ of $\underline{C}$, which was introduced in [5], and its symbol $\Theta_{C, E}$ has the following expansion: For $i=1, \ldots, d$ and $h \in \tilde{\mathcal{H}}$

$$
\Theta_{C, E}\left(D_{E}\right)_{i} h=e_{\emptyset} \otimes\left[\left(D_{C}\right)_{i} h-\gamma D_{*, A} B_{i} h\right]-\sum_{|\alpha| \geq 1} e_{\alpha} \otimes \gamma D_{*, A}\left(A_{\alpha}\right)^{*} B_{i} h
$$

and for $h \in \mathcal{H}^{\circ}$

$$
\begin{aligned}
\Theta_{C, E}\left(D_{E}\right)_{i} h & =-e_{\emptyset} \otimes \gamma D_{*, A} A_{i} h \\
& +\sum_{j=1}^{d} e_{j} \otimes \sum_{\alpha} e_{\alpha} \otimes \gamma D_{*, A}\left(A_{\alpha}\right)^{*}\left(\delta_{j i} I-A_{j}^{*} A_{i}\right) h .
\end{aligned}
$$

Theorem 6.1. Let $U$ and $\tilde{U}$ be unitaries associated with a generalized repeated interaction model, and the lifting $\underline{E}$ of $\underline{C}$ be the corresponding lifting. Then the characteristic function $M_{C, E}$ coincides with the transfer function $\Theta_{U, \tilde{U}}$, i.e.,

$$
\tilde{M}_{\Phi_{C}} \Theta_{U, \tilde{U}}(z)=\Theta_{C, E} \tilde{\Phi}_{E}
$$

Proof. If $h \in \mathcal{H}$ and $i=1, \ldots, d$, then by equation (4.8)

$$
\begin{aligned}
& \tilde{M}_{\Phi_{C}} \Theta_{U, \tilde{U}}(z)\left(F_{i} h z^{\emptyset}\right) \\
= & \tilde{M}_{\Phi_{C}}\left[\tilde{D} z^{\emptyset}+\sum_{\beta \in \tilde{\Lambda}, j=1, \ldots, d} \tilde{C}\left(E_{\bar{\beta}}\right)^{*} F_{j}^{*} z^{\beta j}\right]\left(F_{i} h z^{\emptyset}\right) \\
= & \tilde{M}_{\Phi_{C}}\left[\tilde{D} F_{i} h z^{\emptyset}+\sum_{\beta \in \tilde{\Lambda}, j=1, \ldots, d} \tilde{C}\left(E_{\bar{\beta}}\right)^{*} F_{j}^{*} F_{i} h z^{\beta j}\right] .
\end{aligned}
$$


Case 1. $h \in \tilde{\mathcal{H}}:$

$$
\begin{aligned}
\tilde{D} F_{i} h & =\sum_{j=1}^{d} D_{j} P_{\tilde{\mathcal{H}}} F_{j}^{*} F_{i} h=\sum_{j=1}^{d} D_{j} P_{\tilde{\mathcal{H}}}\left(\delta_{i j} I-E_{j}^{*} E_{i}\right) h \\
& =D_{i} h-\left(\sum_{j=1}^{d} D_{j} P_{\tilde{\mathcal{H}}} E_{j}^{*}\right) E_{i} h=D_{i} h-\tilde{C} E_{i} h \\
& =D_{i} h-\tilde{C}\left(C_{i} h \oplus B_{i} h\right)=D_{i} h-\tilde{C} B_{i} h .
\end{aligned}
$$

Second and last equalities follows from equations (6.5) and (4.13) respectively. By equation (6.5) again we obtain

$$
\begin{aligned}
& \sum_{\beta \in \tilde{\Lambda}, j=1, \ldots, d} \tilde{C}\left(E_{\bar{\beta}}\right)^{*} F_{j}^{*} F_{i} h z^{\beta j} \\
= & \sum_{\beta \in \tilde{\Lambda}, j=1, \ldots, d} \tilde{C}\left(E_{\bar{\beta}}\right)^{*}\left(\delta_{i j} I-E_{j}^{*} E_{i}\right) h z^{\beta j} \\
= & \sum_{\beta \in \tilde{\Lambda}} \tilde{C}\left(E_{\bar{\beta}}\right)^{*} h z^{\beta i}-\sum_{\beta \in \tilde{\Lambda}, j=1, \ldots, d} \tilde{C}\left(E_{\bar{\beta}}\right)^{*} E_{j}^{*} E_{i} h z^{\beta j} \\
= & -\sum_{\beta \in \tilde{\Lambda}, j=1, \ldots, d} \tilde{C}\left(E_{\bar{\beta}}\right)^{*} E_{j}^{*} E_{i} h z^{\beta j} \\
= & -\sum_{\beta \in \tilde{\Lambda}, j=1, \ldots, d} \tilde{C} \tilde{C}\left(E_{\bar{\beta}}\right)^{*}\left(\left(C_{j}^{*} C_{i}+B_{j}^{*} B_{i}\right) h \oplus A_{j}^{*} B_{i} h\right) z^{\beta j} \\
= & \left.-\sum_{\beta \in \tilde{\Lambda}, j=1, \ldots, d} \tilde{C}\left(A_{\bar{\beta}}\right)^{*} A_{j}^{*} B_{i} h z^{\beta j} \quad \text { (by equation }(4.13)\right) \\
= & -\sum_{|\alpha| \geq 1} \tilde{C}\left(A_{\bar{\alpha}}\right)^{*} B_{i} h z^{\alpha} .
\end{aligned}
$$

So by equation $(6.9)$ we have for all $i=1, \ldots, d$ and $h \in \tilde{\mathcal{H}}$

$$
\begin{aligned}
& \tilde{M}_{\Phi_{C}} \Theta_{U, \tilde{U}}(z)\left(F_{i} h z^{\emptyset}\right) \\
= & \tilde{M}_{\Phi_{C}}\left[\left(D_{i} h-\tilde{C} B_{i} h\right) z^{\emptyset}-\sum_{|\alpha| \geq 1} \tilde{C}\left(A_{\bar{\alpha}}\right)^{*} B_{i} h z^{\alpha}\right] \\
= & e_{\emptyset} \otimes \Phi_{C}\left(D_{i} h-\tilde{C} B_{i} h\right)-\sum_{|\alpha| \geq 1} e_{\bar{\alpha}} \otimes \Phi_{C}\left(\tilde{C}\left(A_{\bar{\alpha}}\right)^{*} B_{i} h\right) \\
= & e_{\emptyset} \otimes\left[\left(D_{C}\right)_{i} h-\gamma D_{*, A} B_{i} h\right]-\sum_{|\alpha| \geq 1} e_{\bar{\alpha}} \otimes \gamma D_{*, A}\left(A_{\bar{\alpha}}\right)^{*} B_{i} h .
\end{aligned}
$$

By equation (6.7) it follows that

$$
\begin{aligned}
\tilde{M}_{\Phi_{C}} \Theta_{U, \tilde{U}}(z)\left(F_{i} h z^{\emptyset}\right) & =\Theta_{C, E}\left(e_{\emptyset} \otimes\left(D_{E}\right)_{i} h\right) \\
& =\Theta_{C, E} \tilde{\Phi}_{E}\left(F_{i} h z^{\emptyset}\right) .
\end{aligned}
$$


Case 2. $h \in \mathcal{H}^{\circ}$ :

$$
\begin{aligned}
\tilde{D} F_{i} h & =\sum_{j=1}^{d} D_{j} P_{\tilde{\mathcal{H}}} F_{j}^{*} F_{i} h=\sum_{j=1}^{d} D_{j} P_{\tilde{\mathcal{H}}}\left(\delta_{i j} I-E_{j}^{*} E_{i}\right) h \\
& =D_{i} P_{\tilde{\mathcal{H}}} h-\left(\sum_{j=1}^{d} D_{j} P_{\tilde{\mathcal{H}}} E_{j}^{*}\right) E_{i} h=-\tilde{C} A_{i} h
\end{aligned}
$$

Second equality follows from equation (6.5). By equations (6.5) and (4.13) again we obtain

$$
\begin{aligned}
\sum_{\beta \in \tilde{\Lambda}, j=1, \ldots, d} \tilde{C}\left(E_{\bar{\beta}}\right)^{*} F_{j}^{*} F_{i} h z^{\beta j} & =\sum_{\beta \in \tilde{\Lambda}, j=1, \ldots, d} \tilde{C}\left(E_{\bar{\beta}}\right)^{*}\left(\delta_{i j} I-E_{j}^{*} E_{i}\right) h z^{\beta j} \\
& =\sum_{\beta \in \tilde{\Lambda}, j=1, \ldots, d} \tilde{C}\left(A_{\bar{\beta}}\right)^{*}\left(\delta_{i j} I-A_{j}^{*} A_{i}\right) h z^{\beta j} .
\end{aligned}
$$

So by equation $(6.9)$ we have for all $i=1, \ldots, d$ and $h \in \mathcal{H}^{\circ}$

$$
\begin{aligned}
& \tilde{M}_{\Phi_{C}} \Theta_{U, \tilde{U}}(z)\left(F_{i} h z^{\emptyset}\right) \\
= & \tilde{M}_{\Phi_{C}}\left[-\tilde{C} A_{i} h z^{\emptyset}+\sum_{\beta \in \tilde{\Lambda}, j=1, \ldots, d} \tilde{C}\left(A_{\bar{\beta}}\right)^{*}\left(\delta_{i j} I-A_{j}^{*} A_{i}\right) h z^{\beta j}\right] \\
= & -e_{\emptyset} \otimes \Phi_{C}\left(\tilde{C} A_{i} h\right)+\sum_{\beta \in \tilde{\Lambda}, j=1, \ldots, d} e_{j} \otimes e_{\bar{\beta}} \otimes \Phi_{C}\left(\tilde{C}\left(A_{\bar{\beta}}\right)^{*}\left(\delta_{i j} I-A_{j}^{*} A_{i}\right) h\right) \\
= & -e_{\emptyset} \otimes \gamma D_{*, A} A_{i} h+\sum_{\beta \in \tilde{\Lambda}, j=1, \ldots, d} e_{j} \otimes e_{\bar{\beta}} \otimes \gamma D_{*, A}\left(A_{\bar{\beta}}\right)^{*}\left(\delta_{i j} I-A_{j}^{*} A_{i}\right) h .
\end{aligned}
$$

By equation (6.8) it follows that

$$
\begin{aligned}
\tilde{M}_{\Phi_{C}} \Theta_{U, \tilde{U}}(z)\left(F_{i} h z^{\emptyset}\right) & =\Theta_{C, E}\left(e_{\emptyset} \otimes\left(D_{E}\right)_{i} h\right) \\
& =\Theta_{C, E} \tilde{\Phi}_{E}\left(F_{i} h z^{\emptyset}\right) .
\end{aligned}
$$

Hence we conclude that

$$
\tilde{M}_{\Phi_{C}} \Theta_{U, \tilde{U}}(z)=\Theta_{C, E} \tilde{\Phi}_{E}
$$

The transfer function is a notion affiliated with the input/state/output linear system, while the scattering function is a notion affiliated with the scattering theory in the sense of Lax-Phillips. For our repeated interaction model Theorem 6.1 elucidates that the transfer function is identifiable with the characteristic function of the associated lifting. This establishes a strong connection between a model for quantum systems and the multivariate operator theory. Connections between them were also endorsed in other works like [2, 8], 4] and [10, and this indicates that such approaches to quantum systems using multi-analytic operators are promising. 


\section{References}

[1] J. A. Ball, G. Groenewald, T. Malakorn, Conservative structured noncommutative multidimensional linear systems. The state space method generalizations and applications, 179-223, Oper. Theory Adv. Appl., 161, Birkhäuser, Basel (2006).

[2] B. V. R. Bhat, An index theory for quantum dynamical semigroups, Trans. Amer. Math. Soc., 348 (1996) 561-583.

[3] J. A. Ball, V. Vinnikov, Lax-Phillips scattering and conservative linear systems: a Cuntz-algebra multidimensional setting, Mem. Amer. Math. Soc., 178 (2005).

[4] S. Dey, R. Gohm, Characteristic functions for ergodic tuples, Integral Equations and Operator Theory, 58 (2007), 43-63.

[5] S. Dey,; R. Gohm, Characteristic functions of liftings, J. Operator Theory, 65 (2011), 17-45.

[6] E. Fornasini,; G. Marchesini, Doubly-indexed Dynamical Systems: State Space Models and Structural Properties, Math. Systems Theory, 12 (1978), 59-72.

[7] J. Gough, R. Gohm, Yanagisawa: Linear Quantum feedback Networks, Phys. Rev. A, 78 (2008).

[8] R. Gohm, Noncommutative stationary processes, Lecture Notes in Mathematics, 1839, Springer-Verlag, Berlin (2004).

[9] R. Gohm, Non-commutative Markov chains and multi-analytic operators, J. Math. Anal. Appl., 364 (2010), 275-288.

[10] R. Gohm, Transfer function for pairs of wandering subspaces, Spectral theory, mathematical system theory, evolution equations, differential and difference equations, 385-398, Oper. Theory Adv. Appl., 221, Birkhäuser/Springer Basel AG, Basel, (2012).

[11] B. Kümmerer, H. Maassen, A scattering theory for Markov chains, Infin. Dimens. Anal. Quantum Probab. Relat. Top. 3 (2000), 161-176.

[12] P.D. Lax, R.S. Phillips, Scattering theory, Pure and Applied Mathematics 26 Academic press, New York-London, (1967).

[13] G. Popescu, Isometric dilations for infinite sequences of noncommuting operators, Trans. Amer. Math. Soc., 316 (1989), 523-536.

[14] G. Popescu, Characteristic functions for infinite sequences of noncommuting operators, J. Operator Theory, 22 (1989), 51-71.

[15] G. Popescu, Multi-analytic operators on Fock spaces, Math. Ann., 303 (1995), $31-46$.

[16] G. Popescu, Poisson transforms on some $C^{*}$-algebras generated by isometries, J. Funct. Anal., 161 (1999), 27-61.

[17] G. Popescu, Free holomorphic functions on the unit ball of $\mathcal{B}(\mathcal{H})^{n}$, J. Funct. Anal., 241 (2006), 268-333.

[18] M. Reed, B. Simon, Methods of modern mathematical physics. III. Scattering theory. Academic Press [Harcourt Brace Jovanovich, Publishers], New YorkLondon, (1979).

[19] B. Sz.-Nagy, C. Foias, Harmonic analysis of operators on Hilbert space, North Holland Publ., Amsterdam-Budapest (1970). 
[20] M. Yanagisawa, H. Kimura, Transfer function approach to quantum control, part I: Dynamics of Quantum feedback systems, IEEE Tranactions on Automatic control, 48 (2003), no. 12, 2107-2120.

\title{
Acknowledgment
}

The first author received a support from UKIERI to visit Aberystwyth University, UK in July 2011 which was helpful for this project.

\author{
Santanu Dey \\ Department of Mathematics, \\ Indian Institute of Technology Bombay, \\ Powai, Mumbai- 400076, India \\ e-mail: dey@math.iitb.ac.in \\ Kalpesh J. Haria \\ Department of Mathematics, \\ Indian Institute of Technology Bombay, \\ Powai, Mumbai- 400076, India \\ e-mail: kalpesh@math.iitb.ac.in
}

